\title{
Th1 polarization defines the synovial fluid T cell compartment in oligoarticular juvenile idiopathic arthritis
}

Amélie M. Julé,, Kacie J. Hoyt, ${ }^{1}$ Kevin Wei, ${ }^{2}$ Maria Gutierrez-Arcelus, ${ }^{1,2,3}$ Maria L. Taylor, ${ }^{1}$ Julie Ng, ${ }^{4}$ James A. Lederer, ${ }^{5}$ Siobhan M. Case, ${ }^{1,2}$ Margaret H. Chang, ${ }^{1,2}$ Ezra M. Cohen, ${ }^{1}$ Fatma Dedeoglu, ${ }^{1}$ Melissa M. Hazen, ${ }^{1}$ Jonathan S. Hausmann, ${ }^{1}$ Olha Halyabar, ${ }^{1}$ Erin Janssen, ${ }^{1}$ Jeffrey Lo, ${ }^{1}$ Mindy S. Lo, Esra Meidan, ${ }^{1}$ Jordan E. Roberts, ${ }^{1}$ Mary Beth F. Son, ${ }^{1}$ Robert P. Sundel, ${ }^{1}$ Pui Y. Lee, ${ }^{1,2}$ Talal Chatila, Peter A. Nigrovic, ${ }^{1,2}$ and Lauren A. Henderson ${ }^{1}$

'Division of Immunology, Boston Children's Hospital, Harvard Medical School, Boston, Massachusetts, USA. Division of Rheumatology, Inflammation, and Immunity, Brigham and Women's Hospital, Harvard Medical School, Boston, Massachusetts, USA. ${ }^{3}$ Broad Institute of MIT and Harvard, Cambridge, Massachusetts, USA. ${ }^{4}$ Division of Pulmonary and Critical Care Medicine, and 5Department of Surgery, Brigham and Women's Hospital, Harvard Medical School, Boston, Massachusetts, USA.

Conflict of interest: MBFS received salary support from Childhood Arthritis and Rheumatology Research Alliance (CARRA) and royalties from UpToDate, Inc. PAN has been supported by investigator-initiated research grants from AbbVie, Bristol Myers Squibb, Novartis, Pfizer, and Sobi; consulting from Bristol Myers Squibb, Cerecor, Miach Orthopedics, Novartis, Pfizer, Quench Bio, Sigilon, Simcere, Sobi, and XBiotech; royalties from UpToDate, Inc.; and salary support from CARRA. LAH has received salary support from CARRA and consulting fees from Sobi, Pfizer, and Adaptive Biotechnologies.

Copyright: (c) 2021, Julé et al. This is an open access article published under the terms of the Creative Commons Attribution 4.0 International License.

Submitted: March 2, 2021

Accepted: August 11, 2021

Published: September 22, 2021

Reference information: /CI Insight. 2021;6(18):e149185.

https://doi.org/10.1172/jci.

insight.149185.
Oligoarticular juvenile idiopathic arthritis (oligo JIA) is the most common form of chronic inflammatory arthritis in children, yet the cause of this disease remains unknown. To understand immune responses in oligo JIA, we immunophenotyped synovial fluid T cells with flow cytometry, bulk RNA-Seq, single-cell RNA-Seq (scRNA-Seq), DNA methylation studies, and Treg suppression assays. In synovial fluid, $C D 4^{+}, C D 8^{+}$, and $\gamma \delta$ T cells expressed Th1-related markers, whereas Th17 cells were not enriched. Th1 skewing was prominent in CD4+ $\mathrm{T}$ cells, including Tregs, and was associated with severe disease. Transcriptomic studies confirmed a Th1 signature in $\mathrm{CD4}^{+} \mathrm{T}$ cells from synovial fluid. The regulatory gene expression signature was preserved in Tregs, even those exhibiting Th1 polarization. These Th1-like Tregs maintained Treg-specific methylation patterns and suppressive function, supporting the stability of this Treg population in the joint. Although synovial fluid CD4+ $\mathrm{T}$ cells displayed an overall Th1 phenotype, scRNA-Seq uncovered heterogeneous effector and regulatory subpopulations, including IFN-induced Tregs, peripheral helper T cells, and cytotoxic CD4+ $\mathrm{T}$ cells. In conclusion, oligo JIA is characterized by Th1 polarization that encompasses Tregs but does not compromise their regulatory identity. Targeting Th1-driven inflammation and augmenting Treg function may represent important therapeutic approaches in oligo JIA.

\section{Introduction}

Chronic inflammatory arthritis in children is often considered a single disease by those outside the field of pediatric rheumatology. In fact, juvenile idiopathic arthritis (JIA) is an umbrella term that encompasses 6 disease onset types as defined by the International League of Associations for Rheumatology (ILAR) criteria (1). HLA associations and clinical characteristics further demonstrate that the term JIA includes several distinct diseases, whose underlying biological mechanisms are likely to differ $(2,3)$. Despite this heterogeneity, studies evaluating JIA have often included children with multiple forms of inflammatory arthritis without stratifying by disease type. We sought to focus on the pathogenesis of oligoarthritis, the most common form of JIA in North America (4).

Oligoarticular (oligo) JIA is defined by unique features that include limited joint involvement ( $<5$ joints in the first 6 months of disease) and high rates of uveitis $(1,5)$. In some affected children, chronic arthritis remains restricted to a few joints (persistent oligo JIA), whereas in others it progresses to involve 5 or more joints (extended oligo JIA) (6). Given the limited number of arthritic joints at disease onset, children with oligo JIA are often initially treated with local intraarticular steroid injections instead of systemic immunosuppression. Thus, synovial fluid (SF) samples are easily accessible in this disease, offering an important opportunity to study pediatric autoimmunity at the site of inflammation. 
In oligo JIA, the mechanisms that drive arthritis and result in a persistent or extended disease course are poorly understood. Prior work has uncovered inflammatory CD4 ${ }^{+}$effector T cells (Teffs) in oligo JIA joints. In studies that grouped oligoarthritis with other forms of JIA, Teffs with a Th17 phenotype were identified in the SF, and an inverse relationship between Th17 cells and Tregs was found (7, 8). An increased frequency of SF IL- $17^{+} \mathrm{CD} 4^{+} \mathrm{T}$ cells in oligo JIA has been associated with disease extension (7). In studies restricted to oligo JIA and the closely related seronegative polyarticular JIA, T cells identified in the SF had predominantly Th1 features, including expression of IFN- $\gamma$, which is traditionally considered a Th1 cytokine (9-12). While IFN- $\gamma^{+} \mathrm{CD}^{+} \mathrm{T}$ cells in oligo JIA SF may be bona fide Th1 cells differentiated from naive $\mathrm{CD}^{+} \mathrm{T}$ cells, an alternative hypothesis proposes that these cells are nonclassical Th1 cells derived from Th17 cells (13-15). Nonclassical Th1 cells coexpress Th1 and Th17 markers with respect to cytokines (IFN- $\gamma$, IL-17), chemokine receptors (CXCR3, CCR6), and transcription factors (T-bet, ROR $\gamma \mathrm{t})(16,17)$. Taken together, these past studies highlight the remaining uncertainty over what $\mathrm{T}$ cell populations, Th1, Th17, and/or nonclassical Th1 cells, are implicated pathogenically in oligo JIA.

The interplay between Teffs and Tregs, a subset of $\mathrm{CD}^{+} \mathrm{T}$ cells with suppressive capacity, represents another major area of interest in oligo JIA. In children with JIA, clonal Tregs are found in the SF, suggesting this T cell subset modulates immune responses in the arthritic joint $(18,19)$. Enrichment of Tregs in the SF is associated with fewer cytokine-expressing Teffs and a favorable disease course in oligo JIA (7, 20). Yet, Teffs that escape Treg-mediated suppression have been described in JIA (21-23). Further, the stability of Tregs in the inflammatory environment of the JIA joint is uncertain. Indeed, a proportion of Tregs in JIA SF express low levels of FOXP3, the transcription factor defining the Treg lineage (24). These findings indicate that the dynamics between Tregs and Teffs, as well as the stability of Tregs in the arthritic joint, are likely key factors that determine disease course in oligo JIA. Yet, the mechanisms by which proinflammatory and regulatory $\mathrm{T}$ cells influence the establishment and progression of arthritis in oligo JIA remain poorly understood.

Herein, we sought to determine the immunophenotype of joint-infiltrating $\mathrm{CD} 4^{+} \mathrm{T}$ cells as well as the stability of Tregs in oligo JIA. We showed that $\mathrm{CD} 4^{+} \mathrm{T}$ cells in the SF, including Tregs, were predominantly Th1 polarized. Disease extension was associated with a greater proportion of IFN- $\gamma-$ expressing $\mathrm{CD}^{+} \mathrm{T}$ cells in the joint. Importantly, Th1-skewed SF Tregs in oligo JIA maintained Treg identity and suppressive functionality. Our findings suggest that inhibiting the Th1 pathway and enhancing Treg function may be key in controlling arthritic flares in oligo JIA.

\section{Results}

Patients and samples. A total of 36 patients with oligo JIA, defined by ILAR criteria, provided SF and peripheral blood (PB) samples (Table 1). PB was also obtained from 8 pediatric and 10 adult controls. Details about the samples and experiments they contributed to are provided in Supplemental Table 1 (supplemental material available online with this article; https://doi.org/10.1172/jci.insight.149185DS1).

$C D 4^{+}$T cells in oligo JIA SF adopt a Th1 phenotype. Flow cytometry was used to evaluate the T cell compartment in oligo JIA. Memory $\mathrm{CD}^{+}$, memory $\mathrm{CD}^{+}$, and $\gamma \delta \mathrm{T}$ cells were enriched in oligo JIA joints (Figure $1 \mathrm{~A}$ and Supplemental Figure 1, A and B). The frequencies of $\mathrm{CD} 8^{+}$memory $\mathrm{T}$ (Tmem) cells expressing the Th1 cytokine (IFN- $\gamma$ ) and chemokine receptor (CXCR3) were increased in oligo JIA SF compared with control PB (Supplemental Figure 1A). Similarly, the proportion of $\gamma \delta$ T lymphocytes expressing CXCR3 was higher in SF samples (Supplemental Figure 1B). SF CD4+ Tmem cells showed the most pronounced upregulation of both IFN- $\gamma$ and CXCR3 (Figure 1). On average, $47.8 \% \pm 3.1 \%$ (mean \pm SEM) and $83.9 \% \pm 3.2 \%$ of $\mathrm{CD}^{+}$Tmem cells in SF expressed IFN- $\gamma$ and CXCR3, respectively (Figure 1, B-D). Since intracellular cytokine detection requires stimulation, which leads to CXCR3 downregulation, we were unable to assess for $\mathrm{CXCR}^{+} \mathrm{IFN}-\gamma^{+}$cells. The frequency of $\mathrm{CD} 4^{+} \mathrm{Tmem}, \mathrm{CD} 8^{+} \mathrm{Tmem}$, and $\gamma \delta \mathrm{T}$ cells expressing IL-17 was not increased in the SF compared with the PB (Figure 1, B and C, and Supplemental Figure 1). These results highlight Th1 skewing across T lymphocyte subsets in oligo JIA SF, particularly in CD4 ${ }^{+} \mathrm{T}$ cells.

We characterized $\mathrm{CD}^{+}$Tmem cells with additional flow cytometry studies. Paired $\mathrm{PB}$ and SF samples confirmed enrichment of $\mathrm{CXCR}^{+}$and IFN- $\gamma^{+} \mathrm{CD}^{+}$Tmem cells in oligo JIA joints (Supplemental Figure 2A). To assess for nonclassical Th1 cells that jointly express Th1 and Th17 features, we evaluated the fraction of $\mathrm{CD}^{+} \mathrm{T}$ cells producing IFN- $\gamma$ and IL-17. This population was small $\left(<2 \%\right.$ of $\mathrm{CD} 4^{+}$Tmem in the joint on average) and not significantly increased in JIA SF compared with healthy control PB (Figure 1, B and C). In patients with paired samples, IFN- $\gamma^{+} \mathrm{IL}-17^{+} \mathrm{CD} 4^{+}$Tmem cells were slightly more frequent in the SF $(1.2 \%$ $\pm 0.4 \%$ of $\left.\mathrm{CD}^{+} \mathrm{Tmem}\right)$ than in the $\mathrm{PB}(0.5 \% \pm 0.19 \%)$ (Supplemental Figure $\left.2 \mathrm{~A}\right)$. $\mathrm{T}$ cell stimulation alters 
Table 1. Clinical characteristics of the study participants

\begin{tabular}{|c|c|c|c|}
\hline & Persistent oligo JIA & Extended oligo JIA & $\begin{array}{c}\text { Oligo JIA } \\
\text { (Unknown disease course) }\end{array}$ \\
\hline Sex & 22 female, 3 male & 6 female, 2 male & 2 female, 1 male \\
\hline Age (yrs) at onset & $4.5 \pm 3.2(1-13)$ & $7.9 \pm 5.4(2.5-17)$ & $7.0 \pm 5.6(2-13)$ \\
\hline Active joints at onset & $1.5 \pm 0.9(1-5)$ & $2.0 \pm 1.3(1-4)$ & $1.7 \pm 1.2(1-3)$ \\
\hline Age (yrs) at first sampling & $7.1 \pm 3.7(1.5-15.5)$ & $12.5 \pm 3.7(7-17)$ & $7.5 \pm 5.6(2.5-13.5)$ \\
\hline Active joints at first sampling & $1.6 \pm 1.0(1-5)$ & $1.3 \pm 0.5(1-2)$ & $1.7 \pm 1.2(1-3)$ \\
\hline \multicolumn{4}{|c|}{$\begin{array}{l}\text { Summary values are mean } \pm \text { SD. The range is shown in parentheses. Four patients provided samples at } 2 \text { to } 3 \text { distinct arthritic flares, and } 16 \text { new-onset } \\
\text { oligo IIA patients (within } 6 \text { months of symptom onset) were studied. After sampling, } 2 \text { extended, } 11 \text { remained persistent, and } 3 \text { were lost to follow-up } \\
\text { (unknown disease course). Twenty oligo JIA patients with chronic disease (>6 months' duration) were also studied (14 with persistent and } 6 \text { with extende } \\
\text { oligo JIA). A further } 2 \text { patients with chronic arthritis were later reclassified as psoriatic JIA and enthesitis-related arthritis, respectively (see Supplementa } \\
\text { Table 1). AIncludes } 1 \text { patient with MTX and systemic steroids. ANA positivity was defined by a positive titer at any level on a single occasion. Oligo, } \\
\text { oligoarticular; JIA, juvenile idiopathic arthritis; yrs, years; ANA, antinuclear antibody; ADA, adalimumab; LEF, leflunomide; MTX, methotrexate. }\end{array}$} \\
\hline
\end{tabular}

expression of the Th17-associated chemokine receptor, CCR6; therefore, CD161 was used as an alternative marker of Th17 cells (25). The SF of oligo JIA patients was moderately enriched in CD $161^{+} \mathrm{CD} 4^{+} \mathrm{Tmem}$ cells but at a frequency lower than $\mathrm{CXCR} 3^{+} \mathrm{CD} 4^{+}$Tmem cells (Figure 1, D-F, and Supplemental Figure 2). IFN- $\gamma^{+} \mathrm{CD} 161^{+} \mathrm{CD} 4{ }^{+}$Tmem cells were enriched in the joint, whereas IL- $17^{+} \mathrm{CD} 161^{+} \mathrm{CD} 4^{+}$Tmem cells were not (Figure 1, E and F, and Supplemental Figure 2A). Overall, a large proportion of CD4 ${ }^{+}$Tmem cells in oligo JIA SF expressed IFN- $\gamma$ without IL-17, and over half of CXCR3 ${ }^{+}$cells in the joint did not coexpress CD161, suggesting a substantial population of classical Th1 cells in the joint.

The fraction of IFN $-\gamma^{+} \mathrm{CD} 4^{+}$Tmem cells was higher in the SF of patients with extended $(59.9 \% \pm 2.8 \%)$ versus persistent $(41.6 \% \pm 3.9 \%)$ oligo JIA (Figure $1 G)$. The frequency of IL-17 $7^{+}$IFN- $\gamma^{+} \mathrm{IL}-17^{+}, \mathrm{CXCR}^{+}$, $\mathrm{CD}_{161^{+}}$, and IFN $-\gamma^{+} \mathrm{CD} 161^{+} \mathrm{CD} 4^{+}$Tmem cells did not significantly differ between these 2 groups (Supplemental Figure 2C). The presence of autoantibodies (antinuclear antibody [ANA] positivity) was not associated with differences in the frequency of these $T$ cell populations (Supplemental Figure 2D). SF sampled at disease onset or during a flare of chronic disease showed similar proportions of CD4 ${ }^{+}$Tmem cells expressing CXCR3 and IFN- $\gamma$ (Supplemental Figure 2B). Therefore, the Th1 predominance found in oligo JIA SF persisted throughout the disease course and was associated with severe disease.

$C D 4^{+}$Tregs in oligo JIA SF express Th1 markers. As reported previously, an increased frequency of CD $4^{+} \mathrm{C}$ $\mathrm{D}^{2} 5^{+} \mathrm{CD} 127^{10} \mathrm{FOXP} 3^{+}$cells was observed in oligo JIA SF compared with PB (Figure 2A and Supplemental Figure 3A) (20,22). The SF Treg compartment was depleted in $\mathrm{HELIOS}^{+}$cells, a putative marker of thymically derived Tregs, and SF Tregs expressed high levels of CTLA4 (Figure 2, B-D, and Supplemental Figure 3A) $(26,27)$. A majority of SF Tregs were CXCR3 ${ }^{+}(79.9 \% \pm 3.9 \%)$, in stark contrast with PB Tregs (Figure 2, E and F, and Supplemental Figure 3A). Significantly higher frequencies of CD $161^{+}$Tregs were found in the SF than in the PB (Figure 2, E-G, and Supplemental Figure 3A), but compared with CXCR3 ${ }^{+}$Tregs, the proportion of $\mathrm{CD}_{161^{+}}$Tregs $(9.2 \% \pm 1.2 \%$ ) remained low in the joint. Upon stimulation, $3.6 \% \pm 0.5 \%$ of SF Tregs secreted IFN- $\gamma$, a significant increase compared with Tregs from the PB of patients $(0.9 \% \pm 0.4 \%)$ and pediatric $(0.73 \% \pm 0.17 \%)$ and adult $(0.46 \% \pm 0.08 \%)$ controls (Figure $2, \mathrm{I}$ and $\mathrm{J}$, and Supplemental Figure 3). IL-17-expressing Tregs, which have been identified in systemic JIA, were not found in oligo JIA (28). $\mathrm{CXCR}^{+}$and IFN- $\gamma^{+}$Tregs were found at similar levels in the SF of oligo JIA patients with new-onset and chronic disease, regardless of ANA positivity and disease course (Supplemental Figure 3, B-D). Overall, as in $\mathrm{CD}^{+}$Tmem cells, Tregs with predominantly Th1 features (Th1-like Tregs) were found in oligo JIA SF.

$C D 4^{+} T$ cells in oligo JIA SF express a Th1 transcriptomic signature. To further understand gene expression in $\mathrm{CD}^{+} \mathrm{T}$ cells in oligo JIA, Tregs and Teffs from patients and controls were assessed with bulk RNA-Seq (Figure 3; for interactive interface of transcriptomic results, see https://amjule.shinyapps.io/oligo-JIA/). Principal component analysis (PCA) of the transcriptomic data segregated samples by compartment (PB versus SF) and cell type (Teff versus Treg) (Figure 3A), even for patients receiving methotrexate.

Like flow cytometry analyses, differential gene expression analysis showed that SF Tregs and SF Teffs upregulated IFNG and CXCR3 expression as well as TBX21, which encodes the Th1 lineage-defining 

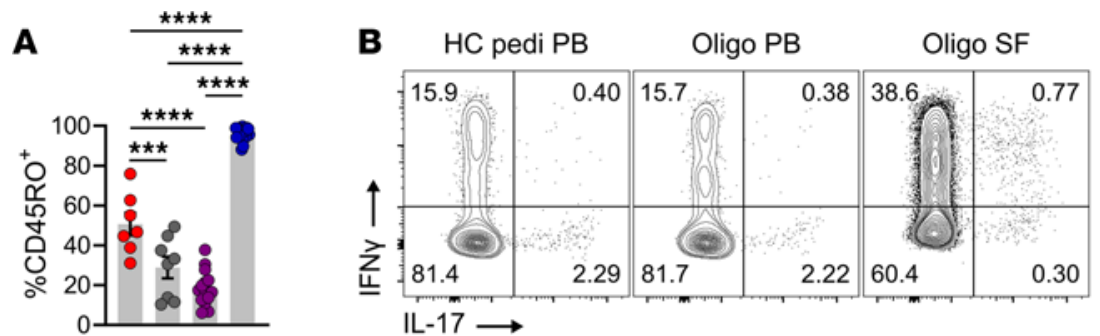

- $\mathrm{HC}$ adult

- $\mathrm{HC}$ pedi

- Oligo PB

- Oligo SF

- Persistent SF

- Extended SF
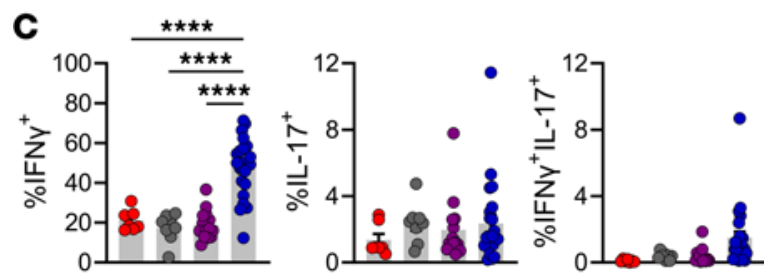

E

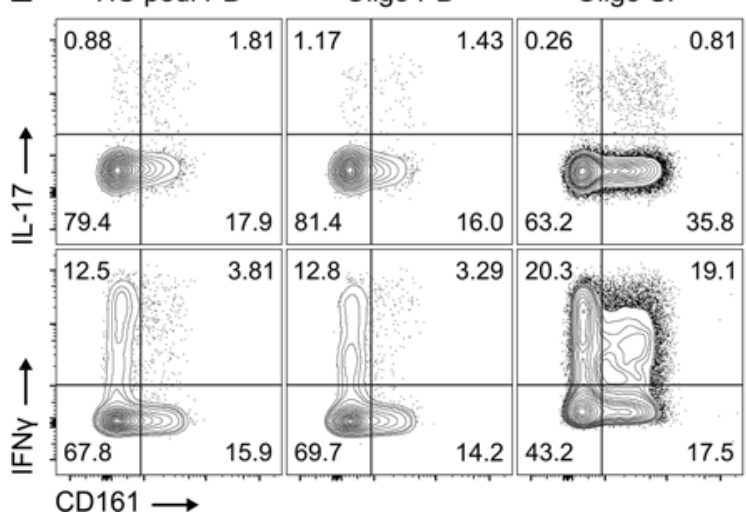

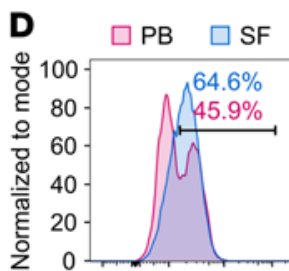

CXCR3

$\mathbf{F}$
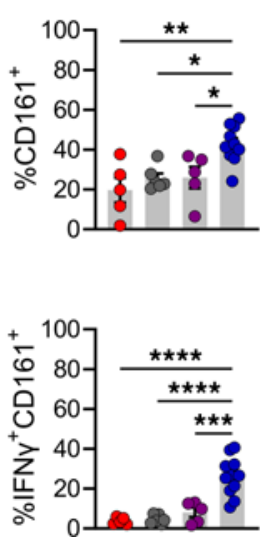
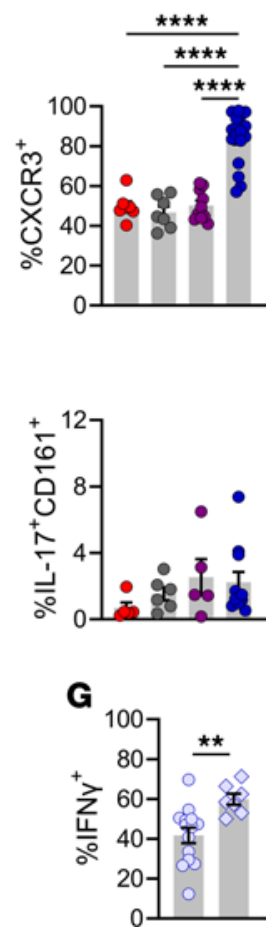

Figure 1. CD4+ memory T cells adopt a Th1 phenotype in the SF of oligo JIA. (A) Percentage of CD45RO+ cells among CD3+CD4+ Iymphocytes. (B) Representative flow staining of cytokine production in stimulated CD3+CD4+CD45RO+ cells (CD4+ Tmem). (C) Percentage of CD4 $4^{+}$Tmem cells expressing IFN- $\gamma$, IL-17, or both after stimulation. The PB of adult $(n=7)$ and pediatric $(n=8)$ controls and PB $(n=14)$ and SF $(n=23)$ of oligo JIA patients were evaluated in A and C. (D) Representative histogram of CXCR3 MFI in paired PB and SF samples from an oligo IIA patient and quantification of CXCR3 ${ }^{+}$cells among unstimulated CD4 $4^{+}$Tmem cells from HC adult PB $(n=7)$, HC pediatric PB $(n=7)$, oligo JIA PB $(n=10)$, and SF $(n=17)$. (E) Representative flow staining of CD161 and cytokine production in stimulated cells gated on CD4+ Tmem cells. (F) Percentage of CD161+ cells (unstimulated) and of CD161 ${ }^{+}$and cytokine dual-expressing cells (stimulated) among CD4+ Tmem cells from HC adult PB $(n=5)$, HC pediatric PB $(n=6)$, oligo JIA PB $(n=5)$, and SF $(n=11)$. (C) Percentage of CD4 ${ }^{+}$ Tmem cells expressing IFN- $\gamma$ in SF samples from persistent $(n=14)$ and extended $(n=7)$ oligo JIA patients. Summary data on bar graphs are mean \pm SEM ${ }^{*} P<0.05,{ }^{* *} P<0.01,{ }^{* *} P<0.001,{ }^{* * *} P<0.0001$. Statistical testing: (A-F) 1-way ANOVA followed by multiple 2-tailed $t$ tests with Tukey's correction;

(C) 2-tailed $t$ test. HC, healthy control; oligo, oligoarticular juvenile idiopathic arthritis; pedi, pediatric; PB, peripheral blood; SF, synovial fluid.

transcription factor T-bet (Figure 3B and Supplemental Data File 1). Gene set enrichment analysis (GSEA) highlighted IFN- $\gamma$ signaling as one of the top enriched gene sets in SF Tregs and SF Teffs compared with PB Tregs and PB Teffs, respectively (Figure 3, C and D). Gene sets related to antigen presentation, T cell receptor (TCR) signaling, and type I IFNs were also enriched in SF Tregs and SF Teffs (Figure 3C, Supplemental Table 4). Th17-related genes were not expressed in SF Tregs at levels significantly higher than in PB Tregs. Transcripts of the Th17 chemokine receptor CCR6 and master gene RORC showed minor increases in SF Teffs compared with PB Teffs, but no differences in IL-17 expression were detected. These results confirm Th1 polarization at the transcriptional level in Tregs and Teffs from the oligo JIA joint.

Oligo JIA SF Tregs maintain robust expression of Treg-related genes. Flow cytometry uncovered cytokineproducing SF Tregs, indicating that these cells may have been reprogrammed to an effector population. To assess for this possibility, we evaluated the transcriptomic signature of Tregs in oligo JIA. Treg-associated transcripts remained significantly elevated in SF Tregs compared with PB Tregs (Figure 3E and Supplemental Data File 1). The Treg transcriptional signature was among the most enriched gene sets in SF Tregs (Figure 3, C and D). Tregs can be thymic in origin ( $\mathrm{tTreg}$ ) or generated in the periphery (pTreg). The low protein 
A

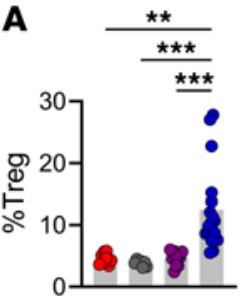

B

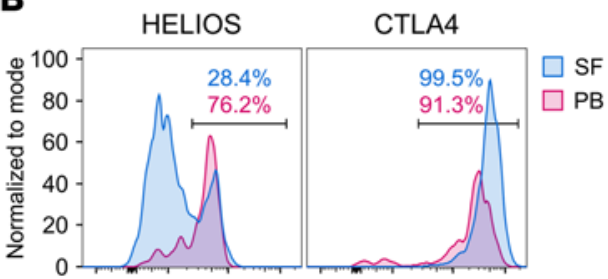

E

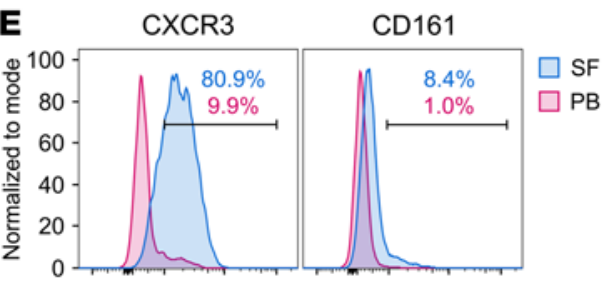

F
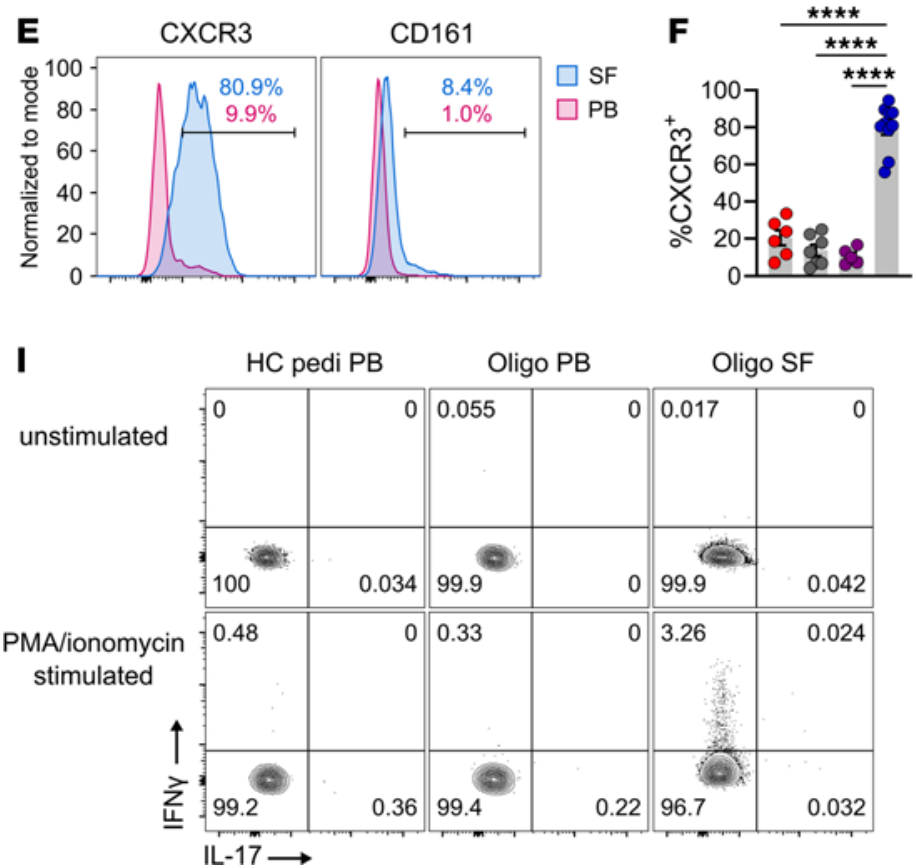
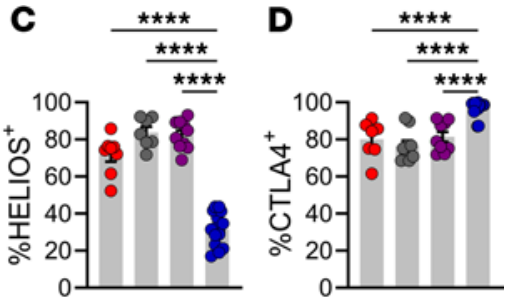

G

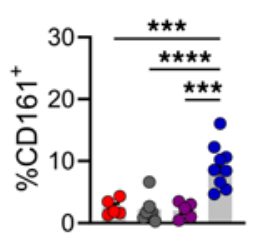

H
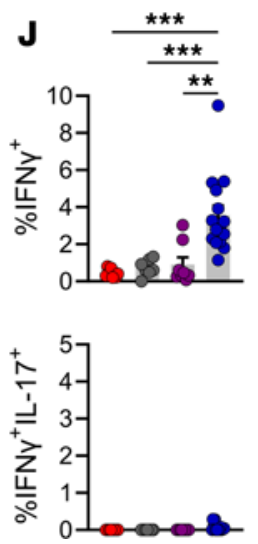
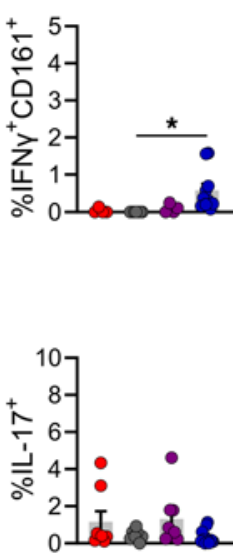

- $\mathrm{HC}$ adult

- $\mathrm{HC}$ pedi

- Oligo PB

- Oligo SF

Figure 2. The SF in oligo JIA patients is enriched in Tregs expressing Th1 markers. (A) Percentage CD2 $5^{+} \mathrm{CD} 127^{\circ} \mathrm{FOPX} 3^{+}$(Tregs) cells among CD4 $4^{+}$lymphocytes in the PB of adult $(n=8)$ and pediatric $(n=7)$ controls and in the PB $(n=10)$ or SF $(n=17)$ of oligo IIA patients. (B) Representative histogram of HELIOS (unstimulated cells) and CTLA4 (stimulated cells) MFI in paired PB and SF samples from an oligo JIA patient, gated on Tregs. (C) Percentage Tregs expressing HELIOS in HC adult PB $(n=8)$, HC pediatric PB $(n=7)$, oligo JIA PB $(n=10)$, and SF $(n=15)$. (D) Percentage Tregs expressing CTLA4 after stimulation in HC adult PB ( $n=$ 7), HC pediatric PB $(n=9)$, oligo JIA PB $(n=9)$, and SF $(n=16)$. (E) Representative histogram of CXCR3 and CD161 MFI in unstimulated Tregs. (F) Percentage Tregs expressing CXCR3 in HC adult PB $(n=6)$, HC pediatric PB $(n=7)$, oligo JIA PB $(n=5)$, and SF $(n=10)$. (C) Percentage Tregs expressing CD161 in HC adult PB ( $n=$ 5), HC pediatric PB $(n=7)$, oligo JIA PB $(n=5)$, and SF $(n=9)$. (H) Percentage Tregs jointly expressing IFN- $\gamma$ and CD161 after stimulation in HC adult PB $(n=4)$, HC pediatric PB $(n=7)$, oligo JIA PB $(n=4)$, and SF $(n=10)$. (I) Representative flow staining of cytokine production in unstimulated and stimulated Tregs. (J) Percentage of Tregs expressing IFN- $\gamma$, IL-17, or both cytokines after stimulation in HC adult PB $(n=8)$, HC pediatric PB $(n=7)$, oligo JIA PB $(n=8)$, and SF $(n=16)$. Summary data on bar graphs are mean \pm SEM. ${ }^{*} P<0.05$, ${ }^{* *} P<0.01$, ${ }^{* *} P<0.001$, ${ }^{* * *} P<0.0001$. Statistical testing: 1-way ANOVA followed by multiple 2-tailed $t$ tests with Tukey's correction. HC, healthy control; pedi, pediatric; oligo, oligoarticular juvenile idiopathic arthritis; PB, peripheral blood; SF, synovial fluid.

expression of HELIOS in SF Tregs documented by flow cytometry suggested pTreg enrichment in the joint; however, the pTreg signature was not found in the transcriptome of SF Tregs (Figure 3E and Supplemental Table 4) (29). Compared with circulating Teffs, SF Teffs upregulated some genes from the Treg transcriptional signature (Figure 3, B-D). This phenomenon is likely secondary to $\mathrm{T}$ cell activation and TCR stimulation, which can induce transient expression of some Treg-related genes (30-33). When SF Tregs were compared directly with SF Teffs, the Treg population retained the highest levels of regulatory gene expression, including FOXP3 (Supplemental Data File 1). Thus, despite increased expression of Th1-related genes, SF Tregs evaluated as a bulk population maintained a regulatory transcriptional program.

Th1-like Tregs in oligo JIA SF maintain features of functional Tregs. We then sought to determine the stability and functionality of Th1-skewed (CXCR $3^{+}$) SF Tregs through methylation studies and suppression assays (Figure 4). CXCR3 ${ }^{+} \mathrm{SF}$ Tregs maintained hypomethylation in the conserved noncoding sequence 2 (CNS2) of FOXP3 and in other Treg-related loci (Figure 4, A and B), as expected for stable Tregs (34). Compared with PB Teffs, SF Teffs displayed significantly lower levels of methylation at FOXP3, CTLA4, and IKZF2 loci, consistent with our transcriptomic findings and the known ability of TCR stimulation to induce epigenetic changes in these genes, even in non-Tregs (35). 
A

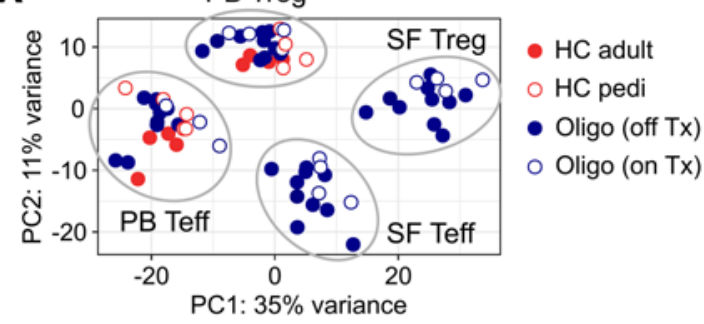

C

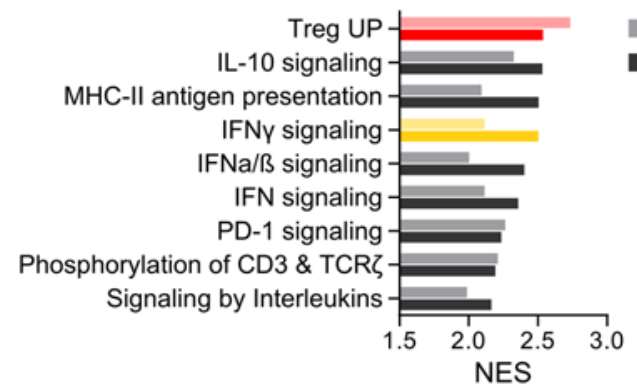

D Treg UP gene set

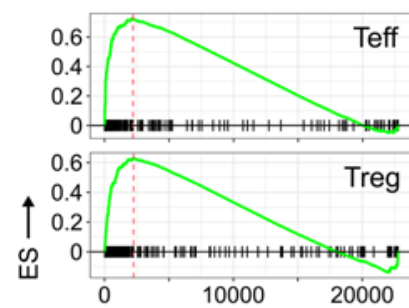

position in LFC-ranked gene list (genes enriched in SF $\rightarrow$ PB)
B IFNy signaling $\quad \bullet$ Treg UP
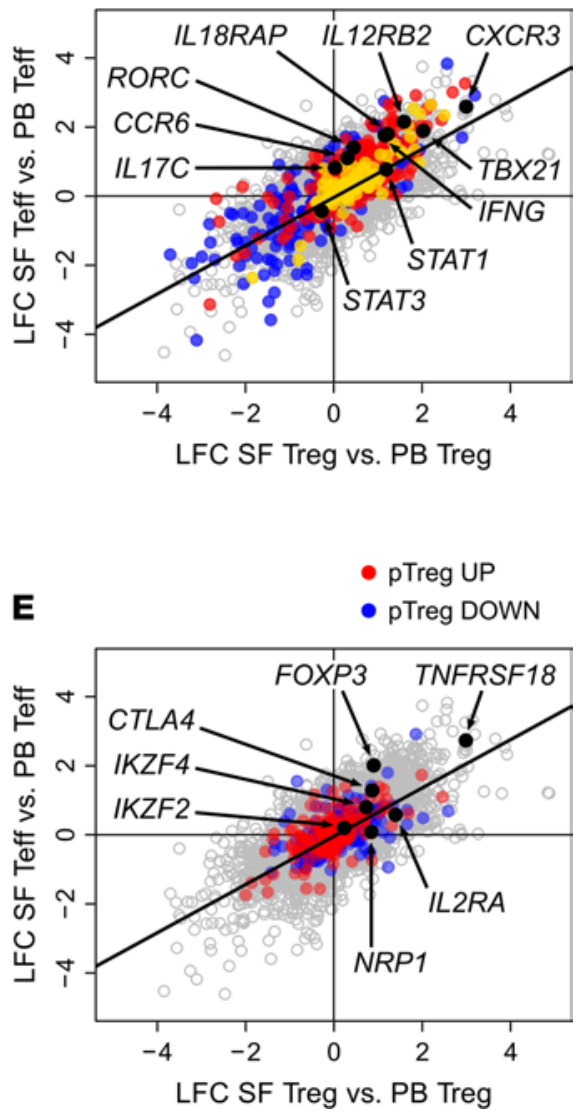

Figure 3. Teffs and Tregs in oligo JIA SF upregulate IFN- $\gamma$-related genes. Tregs (CD4+CD25+CD127 $\left.7^{10}\right)$ and Teffs (CD4+CD25) were sorted from paired SF and PB samples of JIA patients $(n=14)$ and from the PB of pediatric $(n=5)$ and adult $(n=4)$ controls. (A) Principal component (PC) plot based on analysis of complete transcriptomes. (B) Log fold change (LFC) in gene expression derived from independent differential gene expression analyses of SF Tregs versus PB Tregs ( $x$ axis) and SF Teffs versus PB Teffs ( $y$ axis). Genes of the IFN- $\gamma$ signaling pathway (Reactome database) and Treg signature (30) are color-coded. Selected Th1- and Th17-related genes are annotated. (C) Normalized enrichment score (NES) of top hits in gene set enrichment analyses (CSEAs) of Treg and Teff subsets (see Supplemental Table 4 for complete GSEA results). (D) Running enrichment scores for the set of genes upregulated in classical Tregs (30) and for the IFN- $\gamma$ signaling pathway (Reactome database). (E) LFC in gene expression derived from differential gene expression analysis of Treg ( $x$ axis) and Teff ( $y$ axis) subsets. Genes of the peripherally induced Treg ( $p$ Treg) signature (29) are color-coded. Selected Treg-related genes are annotated. $\mathrm{HC}$, healthy control; pedi, pediatric; oligo, oligoarticular juvenile idiopathic arthritis; Tx, treatment.

Importantly, CXCR3 ${ }^{+}$and CXCR3- ${ }^{-}$F Tregs demonstrated a preserved ability to suppress Teff proliferation and cytokine production (Figure 4, C-E, and Supplemental Figure 4). When assessed against Teffs isolated from the PB of different third-party controls, CXCR3 ${ }^{+}$SF Tregs demonstrated an enhanced ability to inhibit Teff proliferation as compared with CXCR3- SF Tregs (Figure 4D and Supplemental Figure 4A). To determine whether SF Teffs are resistant to SF Treg-mediated suppression, autologous SF Teffs were cocultured with both CXCR $3^{+}$and CXCR3- SF Treg subsets. Both CXCR3 ${ }^{+}$and CXCR3- SF Tregs effectively inhibited SF Teff proliferation to a similar degree, which also did not differ from the suppressive capacity of control PB Tregs versus autologous PB Teffs (Figure 4E). Compared with SF Teffs cultured with anti-CD2/ $\mathrm{CD} 3 / \mathrm{CD} 28$ beads alone, the addition of CXCR3 ${ }^{+} \mathrm{SF}$ Tregs significantly decreased the levels of IFN- $\gamma$ in the culture supernatants, indicating a preserved Treg capacity to inhibit cytokine production (Supplemental Figure 4B). Taken together, these bulk-level studies showed stable epigenetic imprinting of the Treg program and preserved suppressive capacity in $\mathrm{CXCR} 3^{+}$and $\mathrm{CXCR} 3^{-} \mathrm{SF}$ Tregs.

Heterogeneity at the single-cell level in Th1-skewed $C D 4^{+} T$ cells in SF. Coexpression of Th1- and Th17-related genes in the same cell is a hallmark of nonclassical Th1 cells that bulk transcriptomic studies cannot unravel. Moreover, the robustness of the Treg transcriptomic signature in the subpopulation of Tregs with Th1 features cannot be determined from bulk RNA-Seq data. Thus, we conducted single-cell RNA-Seq (scRNASeq) and TCR repertoire analysis on sorted Tregs and Teffs from the SF of 2 oligo JIA patients. After quality 
A

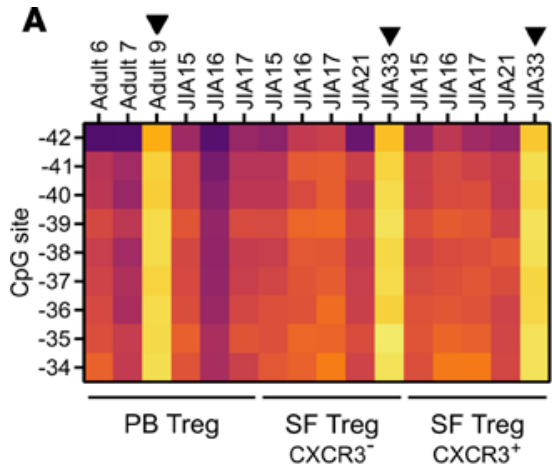

C

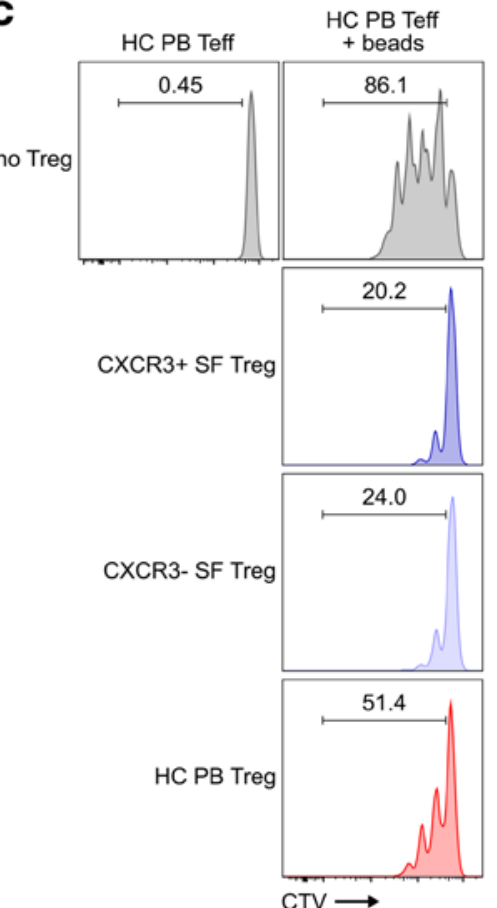

or

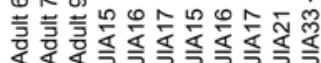

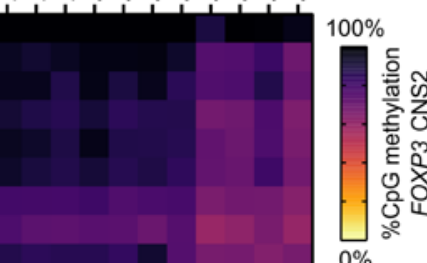

PB Teff SF Teff

D Oligo Treg $\times$ HC Teff

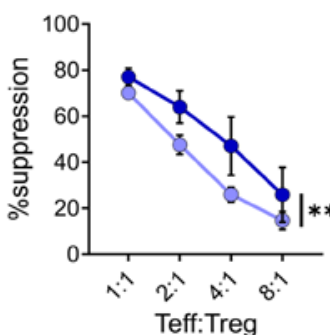

E Treg $x$ autologous Teff

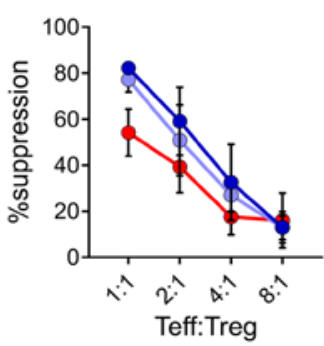

- HC PB Treg

- Oligo SF CXCR3- Treg

- Oligo SF CXCR3+ Treg
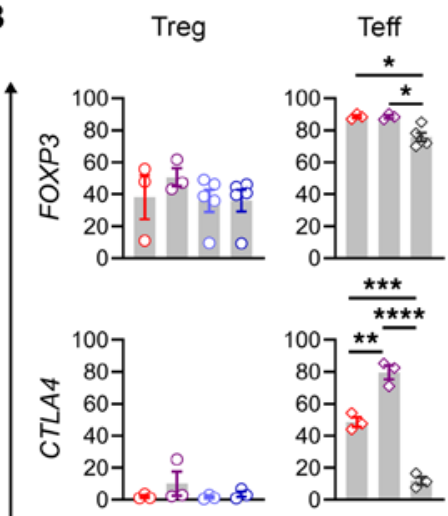

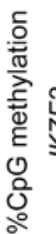
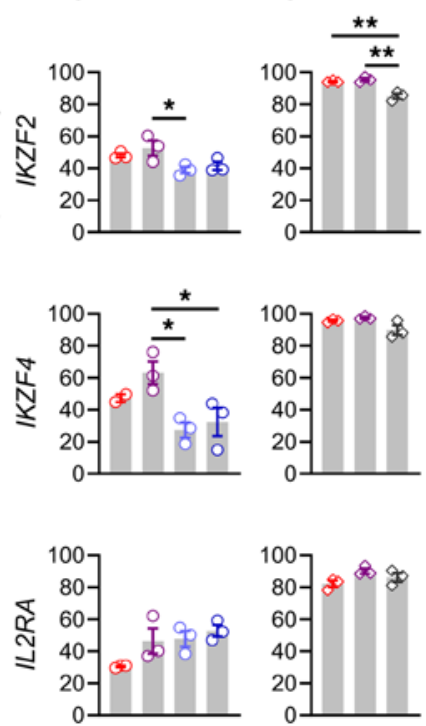

O HC PB Treg

O Oligo PB Treg

$\checkmark$ HC PB Teff

$\diamond$ Oligo PB Teff

O Oligo SF CXCR3- Treg

O Oligo SF CXCR3+ Treg

Figure 4. Th1-like Tregs maintain Treg-specific demethylation patterns and suppressive function. (A) Heatmap of methylation at $9 \mathrm{CpC}$ sites in the conserved noncoding sequence 2 (CNS2) region of the FOXP3 locus in sorted Tregs (CD4+CD25+CD127 $7^{\circ}$ ) and Teffs (CD4+CD25-) from HC adult PB $(n=3)$ and oligo JIA PB $(n=3)$ and SF $(n=5)$. Black arrows highlight male subjects. (B) Percentage methylation (mean \pm SEM) at CpG sites across different Treg-related gene loci in sorted Treg and Teff populations. The location of CpG sites for each locus is specified in Supplemental Table 3. IKZF2 encodes for HELIOS and IKZF4 encodes for EOS. (C) Representative histogram of CTV-positive Teffs (CD4 ${ }^{+}$CD25) after 4 days of coculture alone or with anti-CD2/CD3/CD28 beads and the corresponding Treg $\left(C D 4^{+} C D 25^{+} C D 127^{10}\right)$ population at a ratio of 1:1, gated on dividing Teffs. (D) Percentage suppression (mean \pm SEM) in the proliferation of Teffs from a third-party control after cocultures with CXCR3 ${ }^{+}$SF Tregs or CXCR3- SF Tregs ( $n=4$ controls, $n=7$ oligo lIA patients). See Supplemental Figure 4 for detailed representation per patient. (E) Percentage suppression (mean \pm SEM) in the proliferation of Teffs from HC PB or oligo SF after cocultures with autologous Tregs ( $n=4$ controls, $n=4$ oligo JIA patients). ${ }^{*} P<0.05,{ }^{* *} P<0.01,{ }^{* * *} P<0.001$, ${ }^{* * *} P<0.0001$. Statistical testing: (B) 1-way ANOVA followed by multiple 2-tailed $t$ tests with Tukey's correction; ( $\mathbf{D}$ and $\mathbf{E}$ ) 2-way ANOVA assessing effect of Treg population and Teff/Treg ratio. HC, healthy control; oligo, oligoarticular juvenile idiopathic arthritis; PB, peripheral blood; SF, synovial fluid; CTV, CellTrace Violet.

control, the single-cell data set comprised 1882 Tregs and 4308 Teffs as defined by hashing antibody (Figure 5A). High-dimensional analysis uncovered 14 clusters, including 5 dominated by cells with a Treg profile (clusters 1 to 5; 2004 cells) and 7 dominated by Teffs (clusters 6 to 12; 3955 cells) (Figure 5, B-E; and Figure 6, A and B; https://amjule.shinyapps.io/oligo-JIA/). The final 2 clusters (231 cells) were best characterized by a mitochondrial (cluster 13) or mitotic (cluster 14) signature.

Treg clusters. Five SF Treg clusters were identified at comparable frequencies in both patients (Figure 5D). Key genes associated with the Treg (FOXP3, IL2RA, IKZF2, CTLA4, TNFRSF18) and Th1 (CXCR3, $T B X 21, S T A T 1, I L 12 R B 2)$ profile were detected in all Treg clusters, with differential expression of these and cluster-defining genes across Treg subsets (Figures 5 and 6).

Cluster 1 exhibited robust expression of regulatory genes, including the highest levels of CTLA4, TNFRSF18 (encoding GITR), and the antiinflammatory cytokine IL10. Cluster 1 also had the highest frequencies of cells 

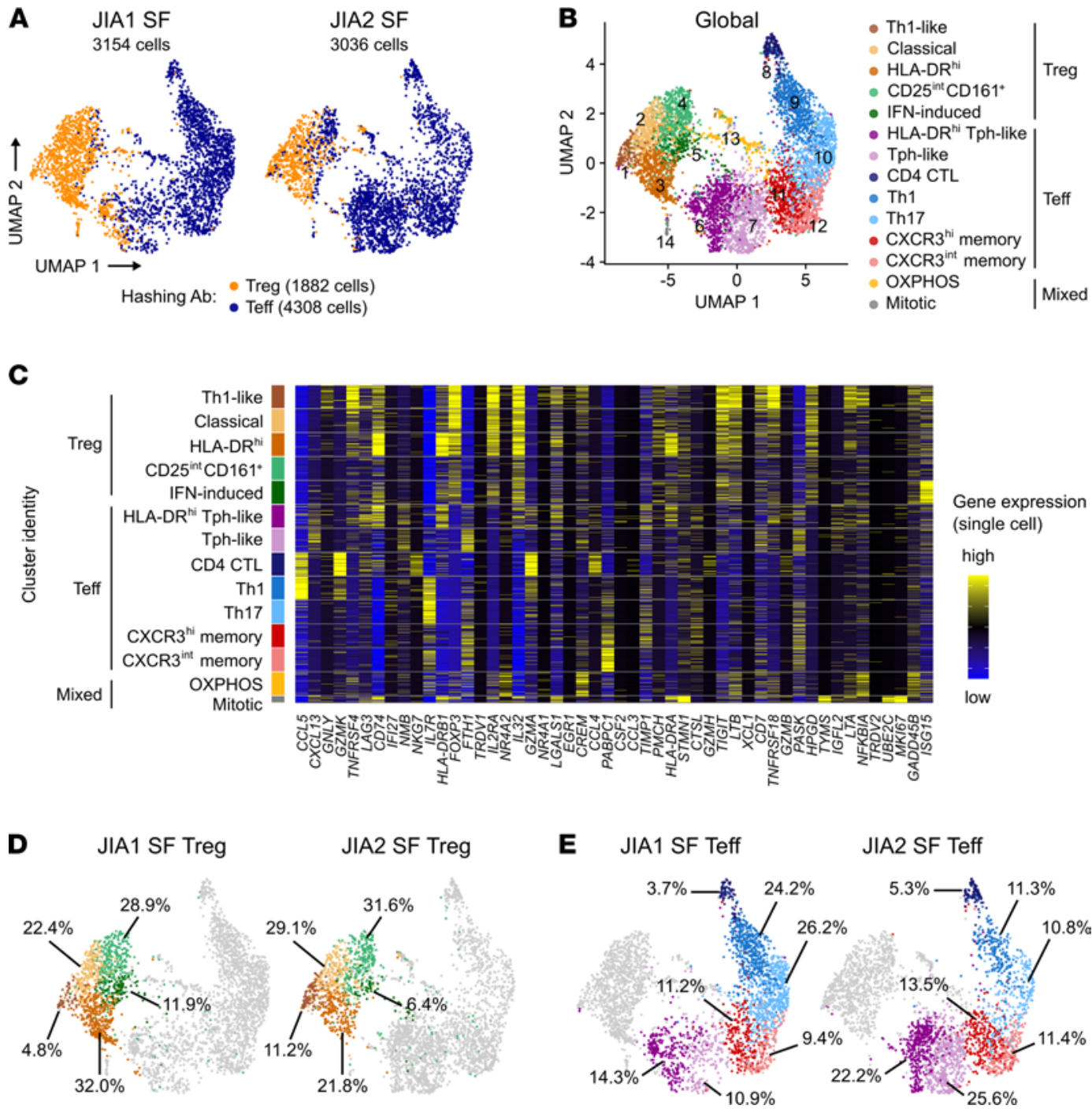

JIA2 SF Teff

Figure 5. ScRNA-Seq reveals heterogeneous subsets of Tregs and Teffs in oligo JIA SF. Sorted Tregs $\left(C D 4^{+} C D 25^{+} C D 127^{\circ}\right)$ and Teffs $\left(C D 4^{+} C D 25^{-}\right)$from the SF of 2 oligo JIA patients were studied with 10x Genomics. (A) Uniform manifold approximation and projection (UMAP) of data set, split by patient and color-coded by hashing antibody. (B) UMAP of global data set (both subjects, 6190 cells), color-coded by cluster. (C) Heatmap showing expression of the top 50 most highly variable genes in 100 randomly selected cells from each cluster. ( $\mathbf{D}$ and $\mathbf{E}$ ) UMAP highlighting cells in Treg (D) and Teff (E) clusters, split by patient and annotated with the corresponding percentage of cells in each cluster. Clusters were defined as 1) Th1-like Treg, 2) classical Treg, 3) activated/ HLA-DR ${ }^{\text {hi }}$ Treg, 4) CD25 ${ }^{\text {int }}$ CD161+ Treg, 5) IFN-induced Treg, 6) activated/HLA-DR ${ }^{\text {hi }}$ T peripheral helper-like (Tph-like), 7) Tph-like, 8) CD4+ cytotoxic T lymphocytes (CTLs), 9) Th1 effector memory, 10) Th17 effector memory, 11) CXCR3 ${ }^{\text {hi }}$ central memory, 12) CXCR3 ${ }^{\text {lo }}$ central memory, 13) cells undergoing OXPHOS metabolism, 14) mitotic cells. JIA, juvenile idiopathic arthritis; SF, synovial fluid.

expressing the Th1-related genes TBX21, IL12RB2, and CXCR3, coupled with the lowest levels of CCR6 and KLRB1 (encoding CD161) observed in any Treg cluster. Detection of $I F N G$ remained rare across the data set, likely because cells were not stimulated prior to scRNA-Seq. These observations led us to define cluster 1 as "Th1-like Tregs" and confirmed that Tregs expressing Th1-related genes maintained a stable Treg transcriptomic signature. Interestingly, cells in this cluster also expressed genes found in $\mathrm{T}$ follicular regulatory cells related to T cell-B cell interactions, including ICOS, $P R D M 1$, and $M A F$, and most markedly, $B A T F(36,37)$.

Clusters 2 (classical Tregs) and 3 (activated/HLA-DR ${ }^{\text {hi }}$ Tregs) were also dominated by cells expressing Treg-related genes at high levels. Cluster 3 stood out for its striking levels of $H L A-D R$ transcripts. Compared with other Treg clusters, cluster 5 (IFN-induced Tregs), and even more so, cluster $4\left(\mathrm{CD} 25^{\text {int }} \mathrm{CD} 161^{+}\right.$Tregs), expressed Treg-related genes at lower levels and included cells harboring a Teff hash, suggesting some cells in these clusters may be peripherally induced Tregs. Cluster 5 was further characterized by upregulation of IFN-induced genes, mostly triggered by type I IFN signaling (STAT1, STAT2, IRF9) and viral sensing (IRF1, IRF3, IRF7, ISG15) or both (38) 
A

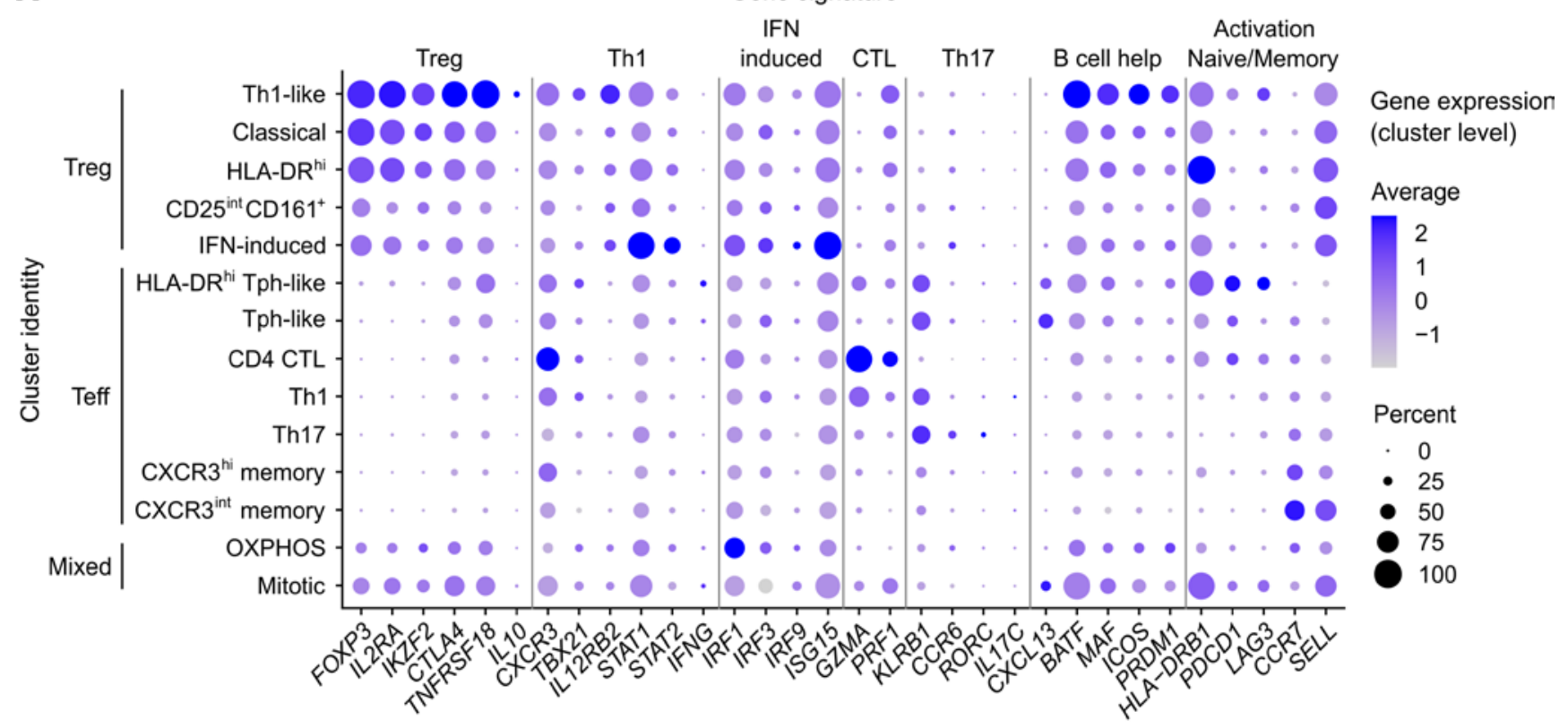

B

Cluster

FOXP3

IL2RA

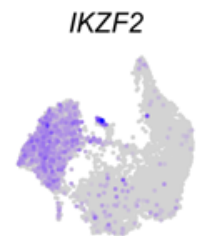

CTLA4

CXCR3

TBX21
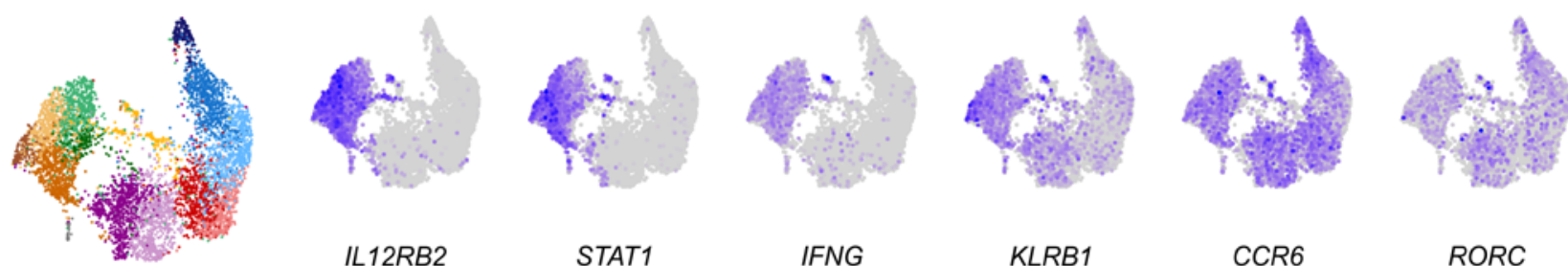

- Th1-like

- Classical

- HLA-DR ${ }^{\text {hi }}$

- $\mathrm{CD} 25^{\text {int }} \mathrm{CD} 161^{+}$

- IFN-induced

- HLA-DR ${ }^{\text {hi }}$ Tph-like
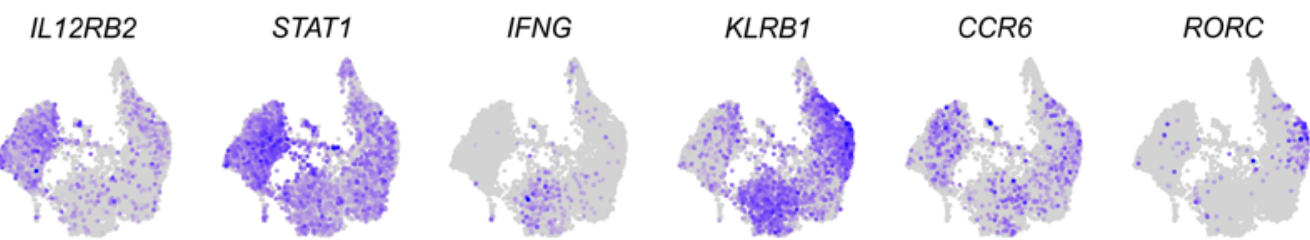

Tph-like

- CD4 CTL

- Th1

- Th17

- $\mathrm{CXCR}^{\text {hi }}$ memory

- CXCR3 ${ }^{\text {int }}$ memory

- OXPHOS

- Mitotic
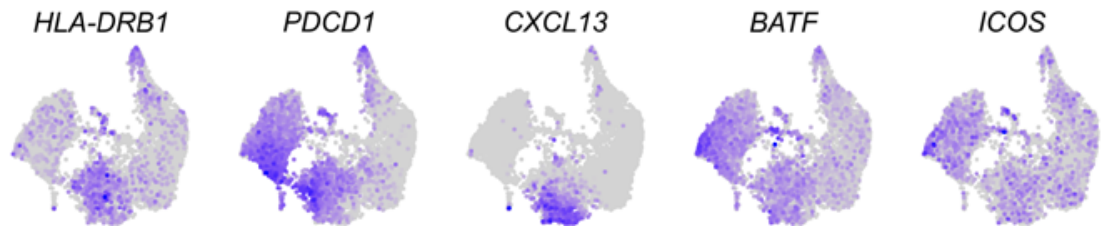

Gene expression (single cell level)

Figure 6. Gene expression in Th1-polarized Tregs and Teffs from oligo JIA SF. Sorted Tregs (CD4+CD25+CD127/0) and Teffs (CD4+CD25-) from the SF of 2 oligo JIA patients were studied with 10x Genomics. (A) Cluster-level expression of markers from key immune cell types (Treg, Th1, Th17, CTLs, Tph/B cell helper T cells), states (activation, exhaustion, naive/memory), and pathways (IFN signaling, viral sensing) used to characterize clusters. For each gene and cluster, the size of the dot is proportional to the percentage of cells expressing the gene in that cluster, and the color code illustrates the average level of expression of all cells in the cluster. (B) Expression levels of key Treg-, Th1-, Th17-, and Tph-related genes across the 6190 cells. The global UMAP color-coded by cluster is shown again for reference. For each gene, cells in the top $95 \%$ quantile of expression level are color-coded; the bottom $5 \%$ appear in gray. Additional genes can be browsed at https://amjule.shinyapps.io/oligo-JIA/. Clusters were defined as 1) Th1-like Treg, 2) classical Treg, 3) activated/HLA-DR hi Treg, 4) CD25intCD161+ Treg, 5) IFN-induced Treg, 6) activated/HLA-DR ${ }^{\text {hi }}$ T peripheral helper-like (Tph-like), 7) Tph-like, 8) CD4+ cytotoxic T lymphocytes (CTLs), 9) Th1 effector memory, 10) Th17 effector memory, 11) CXCR $3^{\text {hi }}$ central memory, 12) $C X C R 3^{\text {lo }}$ central memory, 13) cells undergoing OXPHOS metabolism, 14) mitotic cells.

Teff clusters. scRNA-Seq revealed heterogeneity in oligo JIA SF Teffs, with 7 transcriptionally distinct clusters (Figures 5 and 6). Th1-related genes (TBX21, CXCR3, STAT1) prevailed in Teff clusters. RORC detection was limited overall and largely restricted to cluster 10 (Th17); IL-17 was detected in fewer than 30 cells. Of the 758 Teffs in which TBX21 transcripts were detected, RORC was found in 3.6\%, indicating few cells with features of nonclassical Th1 cells.

Although both patients had cells in all Teff clusters, the abundance of different subpopulations varied between samples (Figure 5E). Most Teffs from JIA1 (51.6\%) had a classical Th1 (cluster 9) or Th17 (cluster 

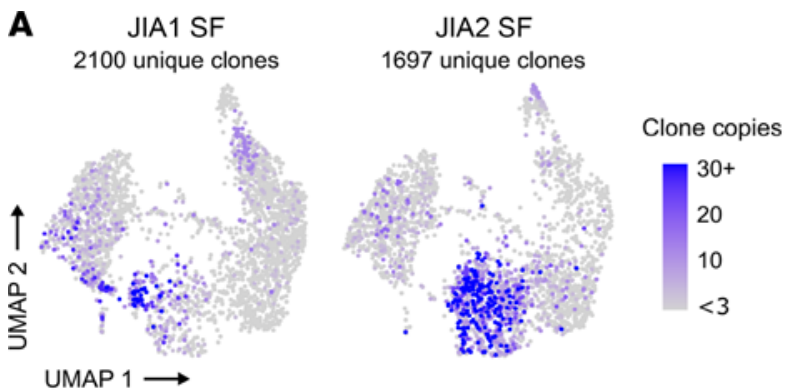

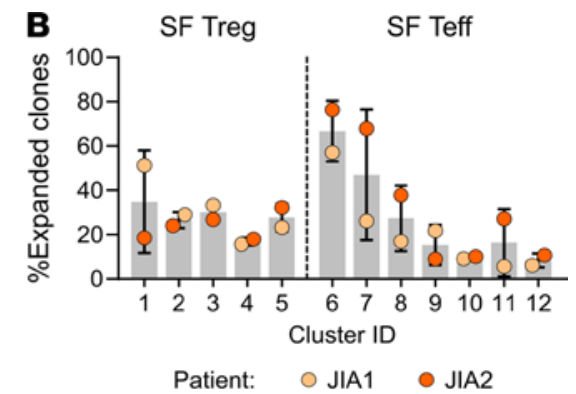

C

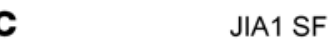

Cluster ID
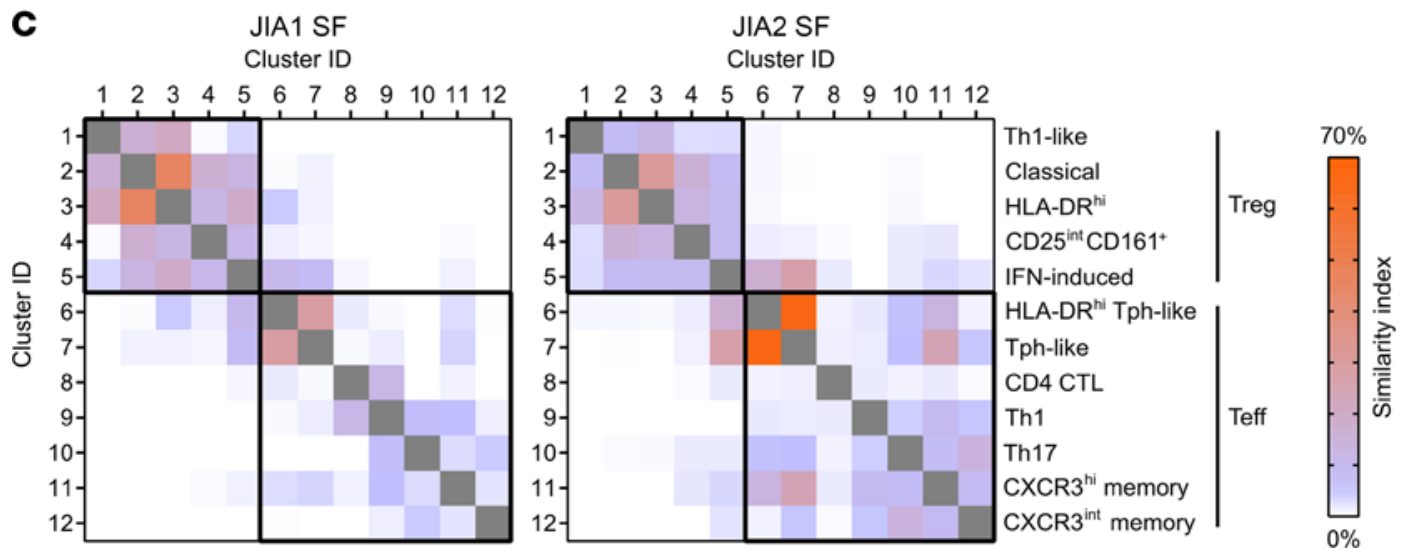

Figure 7. Expanded SF CD4 $4^{+} T$ cell clones primarily concentrate in clusters of Tph-like cells. Sorted Tregs $\left(C D 4^{+} C D 25^{+} C D 127^{\circ}\right)$ and Teffs $\left(C D 4^{+} C D 25^{-}\right)$from the $S F$ of 2 patients with oligo JIA were evaluated at the single-cell level for T cell receptor (TCR) repertoire analysis with 10x Genomics. (A) UMAP projection split by patient, highlighting clones with 3 copies or more across the data set (expanded). A clone is defined by its paired TCR $\alpha$ and TCR $\beta$ chain; cells with missing sequencing data for the $\alpha$ and/or $\beta$ chain were excluded. (B) Percentage (mean \pm SD) of expanded clones ( $\geq 3$ copies) per cluster and study subject. (C) Similarity in clonotypic composition across clusters for each patient measured with the Morisita-Horn index expressed as a percentage. Clusters are defined as follows: 1) Th1-like Treg, 2) classical Treg, 3) activated/HLA-DR ${ }^{\text {hi }}$ Treg, 4) CD25 $5^{\text {int }}$ CD161 ${ }^{+}$Treg, 5) IFN-induced Treg, 6) activated/HLA-DR hi Tph-like, 7) Tph-like, 8) CD4 ${ }^{+}$CTLs, 9) Th1 effector memory, 10) Th17 effector memory, 11) CXCR3 ${ }^{\text {hi }}$ central memory, 12) CXCR $3^{\text {lo }}$ central memory. JIA, juvenile idiopathic arthritis; SF, synovial fluid; Tph, T peripheral helper T lymphocytes; CTLs, cytotoxic T lymphocytes; UMAP, uniform manifold approximation and projection.

10) profile. By contrast, $47.8 \%$ of Teffs from JIA2, who was ANA positive, fell into clusters 6 and 7, characterized by high levels of IFNG along with PDCD1, CXCL13, BATF, and $M A F$, markers of the T peripheral helper (Tph) lineage (36). Markers of T cell activation and exhaustion (HLA-DRB1, PDCD1, LAG3, HAVCR2) were further upregulated in cluster 6 , thus delineated as "activated or HLA-DR ${ }^{\text {hi }}$ Tph-like cells." Other Teff clusters consisted of central memory T cells expressing moderate (cluster 12) to high (cluster 11) levels of CXCR3, accounting for $20 \%-25 \%$ of each patient's SF Teffs. CTLs, expressing granzymes (GZMA, GZMB, GZMK), perforins (PRFI), and cytotoxic granules (NKG7) (cluster 8), accounted for the remaining 3\%-5\% of Teffs.

$T C R$ repertoire. Complete TCR data (paired CDR3 $\alpha$ and CDR3 $\beta$ sequences) were recovered for a total of 5509 cells ( $89 \%$ of the single-cell transcriptomic data set). Clonal expansion was defined as any TCR found in at least 3 sequenced cells; 263 unique clonotypes were expanded (29\% of hashed Tregs and 30\% of hashed Teffs). Highly expanded Teffs concentrated in Tph clusters, and expanded Tregs were identified in multiple Treg clusters (Figure 7, A and B). Clonotypic composition analysis suggested limited similarity between Th1 (cluster 9) and Th17 (cluster 10) cells (similarity index [SI] $=4 \%$ to $6 \%$, Figure 7C). However, the $2 \mathrm{Tph}$ clusters were similarly clonal and dominated by the same clonotypes (SI $=28 \%$ to $65 \%$ ), and so were HLA-DR ${ }^{\text {hi }}$ and classical Tregs $(\mathrm{SI}=31 \%-45 \%)$. Th1-like Tregs were closely related to other Tregs but not to Teffs, supporting their likely natural Treg origin and their stability. By contrast, the repertoire of IFN-induced Tregs was more closely related to the TCRs found in Teffs, especially Tph-like cells.

\section{Discussion}

Our studies in oligo JIA afford the opportunity to better understand autoimmunity in children by evaluating the interplay between regulatory and effector $\mathrm{T}$ cells at the site of active inflammation. In the oligo JIA joint, multiple $\mathrm{T}$ cell lineages expressed markers associated with $\mathrm{Th} 1$ cells; however, the $\mathrm{CD} 4^{+} \mathrm{T}$ cell subset demonstrated the most profound Th1 polarization. This Th1 signature in $\mathrm{CD} 4^{+} \mathrm{T}$ cells was durable and identified at 
disease onset and recurrently with arthritic flares. Enrichment of IFN- $\gamma$-producing CD4 ${ }^{+} \mathrm{T}$ cells in the SF was associated with an extended disease course, suggesting this population may drive severe disease. SF Tregs mirrored the Th1 phenotype of proinflammatory effector $\mathrm{T}$ cells in the joint but retained their regulatory identity.

Previously, increased IL- $17^{+} \mathrm{CD} 4^{+} \mathrm{T}$ cells were reported in JIA SF $(7,8,39,40)$. However, our studies focused on oligo JIA found that only a minority of T cells in oligo JIA SF exhibited Th17 features. This discrepancy likely reflects patient stratification and further demonstrates that JIA encompasses different forms of chronic inflammatory arthritis that are characterized by distinct biology. Our results are concordant with prior work limited to oligo and the closely related seronegative polyarticular JIA, which also highlighted a predominance of cells expressing either CXCR3 or IFN- $\gamma$ in JIA SF (9-12). Expanding on these findings, we demonstrated a robust Th1 gene expression signature in $\mathrm{CD}^{+} \mathrm{T}$ cells found in oligo JIA SF, confirmed persistent Th1 enrichment in the joint longitudinally, and documented an association between the frequency of IFN- $\gamma^{+} \mathrm{CD} 4^{+} \mathrm{T}$ cells in the SF and severe disease.

Our studies provide further clarity on the origin of IFN- $\gamma$-producing CD4 ${ }^{+} \mathrm{T}$ cells in oligo JIA SF and whether classical Th1 (derived from naive CD4 T cells) or nonclassical Th1 cells (derived from Th17 cells) predominate in the joints of children with oligo JIA $(13-16,25)$. Both subsets express the traditional Th1 markers (T-bet, CXCR3, IFN- $\gamma$ ), but nonclassical Th1 cells also retain expression of some Th17 markers (ROR $\gamma \mathrm{t}$, CCR6, CD161, IL-17) $(16,17,41)$. In this study, we found little evidence for coexpression of Th1 and Th17 markers in single cells. Similar to prior findings, IFN- $\gamma^{+} \mathrm{IL}-17^{+}$cells are rare in the joints of oligo JIA patients, with percentages typically less than $3 \%$ of total SF CD4+ Tmem cells (12-14). Further, less than $4 \%$ of T-betexpressing Teffs in scRNA-Seq coexpressed ROR $\gamma \mathrm{t}$, and the repertoire of Th1 and Th17 clusters were not highly related. While we cannot exclude a pathological role for the mixed Th1 and Th17 population, our results indicate that nonclassical Th1 cells represent a small fraction of IFN- $\gamma$-producing cells in oligo JIA SF.

CD161 is a C-type lectin-like receptor that is found on Th17 cells (25). Increased frequencies of IFN- $\gamma^{+} \mathrm{C}$ $\mathrm{D} 161^{+} \mathrm{CD} 4^{+} \mathrm{T}$ cells in oligo JIA SF, found in this study and others, have often been interpreted as an indicator of Th17 origin and a marker of nonclassical Th1 cells $(13,14)$. This interpretation relies on the assumption that CD161 solely indicates Th17 lineage $(13-16,25)$. However, CD161 is expressed on many other T cell types (42). Fergusson et al. proposed that CD161 also defines a population of innate-like T cells poised to produce IFN- $\gamma$ in response to IL-12 and IL-18 (42). In our bulk transcriptomic studies, increased expression of IL-12 and IL-18 receptor was found in SF CD4 ${ }^{+}$T cells. By scRNA-Seq, KLRB1 (encoding CD161) was expressed in virtually all effector memory clusters but was remarkably absent in central memory cells. Both observations support the model put forth by Fergusson et al. and suggest that CD161 is a costimulatory molecule found on CD4 T cells capable of producing IFN- $\gamma$.

SF Tregs in oligo JIA adopt a Th1 phenotype with close to $80 \%$ on average expressing CXCR3 and increased transcription of TBX21 (encoding T-bet). A small but significant fraction of SF Tregs also produce IFN- $\gamma$. Th1-like Tregs have been described previously, although not in JA (43-49). Th1 polarization allows Tregs to accumulate at sites of Th1 inflammation, which is beneficial in circumstances such as infection (46, 48). However, in some inflammatory environments, Th1-like Tregs can convert into effectors that contribute to inflammation $(43,47,49)$. In oligo JIA SF, CXCR3 ${ }^{+}$Tregs maintained their suppressive function and lineage-defining methylation patterns, suggesting preserved Treg identity. These results differ from prior studies that found Teffs from the SF of JIA patients to be resistant to Treg-mediated suppression (21, 22). These discrepant results may be secondary to the population of Tregs cocultured with SF Teffs in suppression assays. Haufe et al. defined Tregs as $\mathrm{CD} 4{ }^{+} \mathrm{CD} 25^{+} \mathrm{T}$ cells, and this population may actually contain activated, nonregulatory $\mathrm{T}$ cells $(21,50)$. While Wehrens and colleagues evaluated the bulk SF Treg population $\left(\mathrm{CD} 4^{+} \mathrm{CD} 25^{+} \mathrm{CD} 127^{10} \mathrm{~T}\right.$ cells), our studies focused on CXCR3 ${ }^{+}$Tregs (22). The data presented herein, as well as the work of others, suggests that $\mathrm{CXCR}^{+}$Tregs in certain environments may have enhanced suppressive function compared with other Treg subsets $(22,46)$. We cannot rule out that the small proportion of IFN- $\gamma^{+}$SF Tregs detected by flow cytometry represents an unstable Treg population. Without prior stimulation, IFNG transcripts in our scRNA-Seq studies remained rare, and the Treg signature of IFN- $\gamma$-producing cells could not be determined. Yet, the Treg signature was preserved in SF Tregs coexpressing other Th1-related genes, suggesting Treg stability in the arthritic joint.

Single-cell immunophenotyping methods have increasingly uncovered the diversity of human Tregs (33, 45, 51). Even in the Th1-polarized environment of oligo JIA SF, we identified several Treg subpopulations, including Th1-like, IFN-induced, HLA-DR ${ }^{+}$, and CD161 ${ }^{+}$Tregs. HLA-DR ${ }^{+}$and CD161 ${ }^{+}$Tregs have been identified previously in JIA and have been shown by others to maintain suppressive capacity, even in inflammatory environments (52-55). Herein, we showed that Th1-like Tregs maintained robust expression of Treg-related 
genes, indicating that this Treg subset also maintains regulatory function in oligo JIA. The population of Tregs displaying a transcriptional fingerprint indicative of viral sensing and IFN signaling is less well defined in the literature, although very similar transcriptomic signatures have been observed in tumor-infiltrating $\mathrm{CD} 4^{+} \mathrm{T}$ cells (56). Further work is needed to understand the functionality of IFN-induced Tregs in oligo JIA.

Single-cell profiling also identified a Teff population with features reminiscent of Tph cells, which were originally described in rheumatoid arthritis synovium and promote B cell responses (36). Interestingly, this Tph-like population was found at higher frequency and clonality in the ANA-positive patient. This aligns with the recent observation of an increased frequency of $\mathrm{CD} 4^{+} \mathrm{T}$ cells that produce the Tph-related cytokine IL-21 in ANA-positive JIA patients (12). Yet, the ability of Tph-like cells in JIA SF to promote B cell maturation and pathological autoantibody production remains unknown. In addition, a cluster of $\mathrm{CD} 4^{+} \mathrm{T}$ cells expressing multiple markers of cytotoxicity, which are most commonly associated with $\mathrm{NK}$ and $\mathrm{CD} 8^{+} \mathrm{T}$ cells, was uncovered. While still debated, it has been proposed that CD4 ${ }^{+}$CTLs kill cells in an MHC class II-restricted manner (57). CD4 ${ }^{+}$CTLs have been most commonly identified in the context of viral infection or antitumor immunity and appear to be closely related to Th1 cells $(58,59)$. Interestingly, scRNA-Seq studies in patients with COVID-19 identified a similar population of CD4+ CTLs alongside cytotoxic Tfh cells; and the abundance of these cytotoxic populations correlated with limited Treg responses and severe disease $(60,61)$. The identification of $\mathrm{CD}^{+}$CTLs in oligo JIA suggests that this population of $\mathrm{CD}^{+} \mathrm{T}$ cells may be important in Th1-mediated autoimmunity, particularly in inflamed peripheral tissues.

Our human-based research has limitations, which we attempted to address by evaluating a well-defined study group (oligo JIA) and accounting for disease duration and treatment. Although every effort was made to focus on oligo JIA, not all patients underwent testing for HLA-B27 and it is possible that some children with enthesitis-related arthritis were included in this study. The young age of onset and female predominance in this cohort is a characteristic of oligo JIA and suggests that most patients were classified correctly. These studies were performed with T cells obtained from the SF, which may not fully represent the T cell populations in synovial tissue. Synovial biopsies are not routinely performed in children with oligo JIA, and our access to such tissue samples was limited. Similarly, our studies of Treg function were restricted to in vitro suppression assays that measure Teff proliferation and cytokine production and may not reflect the complex interactions that occur in vivo between Tregs and other non-T cell populations. In addition, only 2 patients were evaluated by scRNA-Seq, but these results were interpreted alongside data derived from a larger patient cohort.

Our study provides important insights into the $\mathrm{CD} 4^{+} \mathrm{T}$ cells that contribute to Th1 responses in oligo JIA. Our results suggest that controlling Th1-mediated inflammation, potentially via Treg-based approaches delivered to the joint, may be an important strategy to restore tolerance in oligo JIA.

\section{Methods}

Patients and samples. A board-certified pediatric rheumatologist reviewed the medical records to gather clinical data and confirm the diagnosis. Patients within 6 months of symptom onset were classified as newonset oligo JIA. ANA positivity was defined by a positive titer at any level on a single occasion. PB and/ or discarded SF samples were collected from patients with oligo JIA defined by the ILAR criteria (1). As outlined in the ILAR criteria, children with enthesitis, known HLA-B27 positivity, psoriasis, and/or family history of psoriasis in a first-degree relative at the time of enrollment and sample collection were excluded. When available, medical records were reviewed prior to publication of this study to identify interval changes in the clinical history of enrolled study subjects and determine whether the JIA classification had changed. PB samples were also obtained from controls. Clinical characteristics of the study cohort are available in Table 1 and Supplemental Table 1.

Cell isolation. Mononuclear cells were isolated from the PB and SF by Ficoll density gradient centrifugation (GE Healthcare, now Cytiva), cryopreserved in liquid nitrogen, and subsequently thawed into RPMI (HyClone) with 10\% FBS (MilliporeSigma), penicillin $100 \mathrm{IU} / \mathrm{mL}$ (Corning Cellgro), streptomycin $100 \mu \mathrm{g} /$ $\mathrm{mL}$ (Corning Cellgro), nonessential amino acids (Corning Cellgro), sodium pyruvate $1 \mathrm{mM}$ (Corning Cellgro), and HEPES $10 \mathrm{mM}$ (Thermo Fisher Scientific). After thawing, PBMCs and synovial fluid mononuclear cells (SFMCs) were incubated overnight before downstream experiments.

Flow cytometry. PBMCs and SFMCs were left unstimulated or stimulated with a cocktail of PMA, ionomycin, and brefeldin A (BD Biosciences) for 4 hours. Cells were stained for viability and surface markers, then fixed and permeabilized (eBioscience) before intracellular staining. Antibodies and dyes are listed in Supplemental Table 2. Data were acquired on an LSRFortessa (BD Biosciences) and analyzed using FlowJo. 
Bulk RNA-Seq. After enrichment for $\mathrm{CD} 4^{+} \mathrm{T}$ cells (Miltenyi Biotec), Tregs $\left(\mathrm{CD} 3^{+} \mathrm{CD} 4^{+} \mathrm{CD} 25^{+} \mathrm{CD} 127^{10}\right.$ ) and Teffs $\left(\mathrm{CD}^{+} \mathrm{CD}^{+} \mathrm{CD} 25^{-}\right)$were isolated by FACS. RNA was extracted from 10,000 cells of each population (QIAGEN) and sequenced using the Smart-seq2 platform at Broad Technology Labs (62). Sequencing was run in 2 batches on an Illumina NextSeq500 sequencer, with both runs relying on identical pipelines.

Samples were analyzed and quality-controlled as previously described (28). Briefly, the bcbio-nextgen RNA-Seq analysis pipeline was used (https://bcbio-nextgen.readthedocs.org/en/latest/). Trimmed reads that passed quality control with FastQC (http://www.bioinformatics.babraham.ac.uk/projects/fastqc/) were aligned to UCSC build GRCh37 of the human genome (Homo sapiens), augmented with transcript information from Ensembl release 90 using STAR (63). Counts of reads aligning to known genes were computed using featureCounts, and transcripts per million (TPM) measurements per isoform were determined through quasi-alignment using Salmon $(64,65)$. Counts per gene estimated by tximport from Salmon quasi-alignments were used for downstream analyses in R version 3.6.2 (https://www.R-project.org/).

To determine the impact of running samples in 2 batches, PCA was performed on full gene expression profiles after alignment and scrutinized for association with various covariates, including subject characteristics and technical factors. The PCA uncovered a minor technical batch effect, which was addressed by regressing out the sequencing run variable when modeling the data and estimating $\log _{2}$ fold changes in differential gene expression analysis. Two patients whose diagnosis evolved from oligoarticular to enthesitisrelated arthritis or psoriatic JIA contributed paired samples to this transcriptomic study. In PCA, samples from these patients did not cluster apart from other JIA samples obtained from the same compartment (PB or $\mathrm{SF}$ ); therefore, to maximize the statistical power of our analysis, the patients with enthesitis-related arthritis and psoriatic JIA were not excluded. Similarly, patient and control PB samples did not cluster apart in PCA; therefore, transcriptomic analyses of PB T cell populations jointly considered JIA and control subjects.

Differential gene expression analysis was run using DESeq2 version 1.22.2 (66). DESeq2-estimated $\log _{2}$ fold changes in gene expression were corrected using the normal method implemented in the lfcShrink function (66). Rank-corrected $\log _{2}$ fold change values served as input for a cutoff-free GSEA using clusterProfiler version 3.14.3 (67). GSEA compared sequencing data with the Reactome database (immune system and metabolism subsets), the human Treg gene set, and the mouse peripheral/induced Treg gene set $(29,30,68)$.

Treg suppression assays and $C p G$ methylation studies. For these analyses, Teffs ( $\left.\mathrm{CD} 3^{+} \mathrm{CD} 4^{+} \mathrm{CD} 25^{-}\right)$as well as bulk or $\mathrm{CXCR}^{+}$and $\mathrm{CXCR} 3^{-}$Tregs $\left(\mathrm{CD} 3^{+} \mathrm{CD} 4^{+} \mathrm{CD} 25^{+} \mathrm{CD} 127^{10}\right)$ were isolated using FACS. The suppressive capacity of SF Tregs was assessed against autologous SF Teffs as well as against PB Teffs from third-party controls; the suppressive capacity of control PB Tregs was assessed against autologous PB Teffs. Teffs were labeled with CellTrace Violet (Invitrogen), stimulated with anti-CD2/CD3/CD28 beads (Miltenyi Biotec), and cocultured with Tregs at various ratios. After 4 days, Teff cell division was assessed by dye dilution using flow cytometry (LSRFortessa, BD Biosciences), and cytokine concentrations in culture supernatants were measured by Luminex FlexMap 3D assay system per the manufacturer's instructions. For methylation analysis, cells were pelleted and snap-frozen. EpigenDx extracted DNA and performed the bisulfite conversion pyrosequencing for methylation analysis of the cytosine guanine dinucleotide sites listed in Supplemental Table 3.

scRNA-Seq with TCR repertoire. First, 5000 Tregs and 5000 Teffs were FACS-sorted from the SF of 2 patients as for bulk RNA-Seq, stained with hashing antibodies, and pooled together for processing in a single batch (69). After encapsulation and barcoding (10x Genomics), cells were lysed, and cDNA was prepared to create a $5^{\prime}$ gene expression library; a variable, diversity, and joining gene-enriched library for TCR repertoire analysis; and a hashtag oligonucleotide library for source sample identification (70). Libraries were sequenced using Illumina NovaSeq 6000. Demultiplexing and gene alignment were performed with CellRanger (10x Genomics) (70).

After gene alignment, the complete matrix of raw features generated by CellRanger was imported in $\mathrm{R}$ version 3.6.2 using the Read10X function of Seurat version 3.1.5 (71). Droplets with fewer than 500 genes, fewer than 800 unique molecular identifiers (UMIs), a $\log _{10}$ (gene) to $\log _{10}$ (UMI) ratio less than 0.85 , or a mitochondrial to genomic transcript ratio more than than 0.15 were excluded, leaving a total of 7304 cells. Genes detected in 10 cells or less were filtered out, leaving a total of 13,772 genes. Hashing data were normalized using centered log ratios, then demultiplexed using Seurat's HTODemux function with the default "clara" method and a positive quantile cutoff set to 0.98 . Cells with unknown or multiple hash detection were excluded at this stage, leaving a total of 6190 cells for analysis.

The transcriptomic data that passed all quality control steps above were normalized with Seurat's SCTransform function, using patient ID as a variance stabilization covariate to limit clustering by patient and searching for the top 3200 most variable features in the data set. To enable clustering by transcriptomic profile 
regardless of clonal identity, the list of most variable features was manually filtered out for TRAV, TRAJ, $T R B V$, and TRBJ genes, then reduced to a length of 3000 features.

PCA was run on the resulting filtered list of top 3000 most variable features. Beyond the 11th principal component (PC), each new PC contributed little more to explaining the overall variation in the data set. We therefore used 11 PCs for Louvain clustering analysis with Seurat's FindNeighbors and FindClusters functions and settled on a resolution of 1.0 to define the clusters in the data set. All visualizations of the single-cell data were based on a uniform manifold approximation and projection (UMAP) dimensionality reduction, computed with uwot version 0.1 .5 and using 22 PCs.

For cluster characterization, we used Seurat's FindMarker function alongside a manual search for genes of interest. Unless results from the marker search highlighted another profile (notably, high expression of genes suggestive of a specific metabolic or mitotic cell state), we sought to categorize clusters as presenting a predominately Treg or Teff transcriptomic profile. We thus defined clusters with simultaneous expression of FOXP3, IL2RA, IKZF2, CTLA4, and TNFRSF18 at moderate to high levels as "Treg clusters." In contrast, clusters with low to no detection of these key Treg genes were defined as "Teff clusters." The Treg versus Teff identity assignment was further informed by the relative proportion of cells hashed with a Treg or Teff antibody within the corresponding cluster, although a small fraction of cells with a Treg hash clustered with cells presenting a Teff transcriptomic profile and vice versa. For further characterization of Treg and Teff clusters, we qualitatively contrasted their gene expression patterns with known markers of different $\mathrm{T}$ cell states (including Th1, Th17, T follicular helper cells, and activated/circulating lymphocytes).

For the TCR repertoire component, we corrected the raw copy number of each clonotype identified by CellRanger vdj to account solely for the 6190 cells retained in prior steps of the analysis. TCR repertoire characteristics were studied using custom $\mathrm{R}$ code and stored in the metadata slot of the Seurat object. The similarity in clonotypic composition between 2 clusters is defined as 1-MH, where $\mathrm{MH}$ is the Morisita-Horn dissimilarity index calculated using the vegdist function of the vegan $\mathrm{R}$ package version 2.5.7 (72).

Data accessibility. Raw bulk and scRNA-Seq as well as TCR repertoire data are available at ImmPort (https://www.immport.org) under study accession SDY1777. An interactive tool for exploration of the analyzed data sets is available at https://amjule.shinyapps.io/oligo-JIA/.

Statistics. One-way ANOVA with Tukey's correction for multiple comparisons was used to compare differences across more than 2 study groups for normally distributed data. Paired or unpaired 2-tailed $t$ tests were used for analyses restricted to 2 study groups with normally distributed data. Significance was defined as a $P$ value less than 0.05. For GSEA, significance was defined at an FDR ( $q$ value) less than 0.05 .

Study approval. These studies were approved by the IRB at Boston Children's Hospital (IRB protocols 07-09-0375 and IRB-P00005723). Written informed consent (and assent when appropriate) was provided by participants and/or legal guardians.

\section{Author contributions}

LAH and AMJ conceived and designed the study. LAH supervised the research. PAN and TC contributed to the experimental design. AMJ, KJH, MT, JN, JAL, and LAH performed the experiments and acquired the data. KW and MGA assisted in the scRNA-Seq experiments and analysis. KJH, MT, SMC, MHC, EMC, FD, MMH, JH, OH, EJ, JL, MSL, EM, JER, MBFS, RPS, PYL, PAN, and LAH recruited patients and collected samples. AMJ, KJH, JN, and LAH analyzed the data. AMJ and LAH drafted the manuscript with input from PAN and PYL. All authors edited the manuscript and approved the final version.

\section{Acknowledgments}

We thank the patients and volunteers for participation in this study. We thank Steve Moskowit for support in preparing the graphical abstract for this study. The graphical abstract makes use of images available at Servier Medical Art (https://smart.servier.com). AMJ was supported in part by a microgrant from the Joint Biology Consortium (P30 AR070253). KW is supported by NIH National Institute of Arthritis and Musculoskeletal and Skin Diseases (NIAMS) K08 AR077037. JN was funded by T32 HL007633-35. JER was supported by the NIH grant 5T32AI007512-34. PAN was funded by NIAMS awards R01 AR075906, R01 AR073201, P30 AR070253; the Fundación Bechara; and the Arbuckle Family Fund for Arthritis Research. LAH was funded by NIAMS K08 AR073339, NIAMS P30 AR070253, an Investigator Award of the Rheumatology Research Foundation, an All Arthritis grant from the Arthritis National Research Foundation, and a Career Development Award from the Office of Faculty Development at Boston Children's Hospital. 
Address correspondence to: Lauren A. Henderson, 1 Blackfan Circle, Karp Family Research Building, 10 ${ }^{\text {th }}$ floor, Boston, Massachusetts 02115, USA. Phone: 617.919.2764; Email: Lauren.Henderson@childrens.harvard.edu. KJH's present address is: Virginia Tech Carilion School of Medicine, Roanoke, Virginia, USA.

1. Petty RE, et al. International League of Associations for Rheumatology classification of juvenile idiopathic arthritis: second revision, Edmonton, 2001. J Rheumatol. 2004;31(2):390-392.

2. Hinks A, et al. Fine-mapping the MHC locus in juvenile idiopathic arthritis (JIA) reveals genetic heterogeneity corresponding to distinct adult inflammatory arthritic diseases. Ann Rheum Dis. 2017;76(4):765-772.

3. Nigrovic PA, et al. Review: genetics and the classification of arthritis in adults and children. Arthritis Rheumatol. 2018;70(1):7-17.

4. Oen KG, Cheang M. Epidemiology of chronic arthritis in childhood. Semin Arthritis Rheum. 1996;26(3):575-591.

5. Saurenmann RK, et al. Prevalence, risk factors, and outcome of uveitis in juvenile idiopathic arthritis: a long-term followup study. Arthritis Rheum. 2007;56(2):647-657.

6. Guillaume S, et al. Long-term outcome and prognosis in oligoarticular-onset juvenile idiopathic arthritis. Arthritis Rheum. 2000;43(8):1858-1865

7. Nistala $\mathrm{K}$, et al. Interleukin-17-producing T cells are enriched in the joints of children with arthritis, but have a reciprocal relationship to regulatory T cell numbers. Arthritis Rheum. 2008;58(3):875-887.

8. Olivito B, et al. Th17 transcription factor RORC2 is inversely correlated with FOXP3 expression in the joints of children with juvenile idiopathic arthritis. J Rheumatol. 2009;36(9):2017-2024.

9. Gattorno M, et al. Synovial fluid T cell clones from oligoarticular juvenile arthritis patients display a prevalent Th1/Th0-type pattern of cytokine secretion irrespective of immunophenotype. Clin Exp Immunol. 1997;109(1):4-11.

10. Issekutz AC, et al. Coexpression of chemokine receptors CCR5, CXCR3, and CCR4 and ligands for P- and E-selectin on T lymphocytes of patients with juvenile idiopathic arthritis. Arthritis Rheum. 2011;63(11):3467-3476.

11. Wedderburn LR, et al. Selective recruitment of polarized T cells expressing CCR5 and CXCR3 to the inflamed joints of children with juvenile idiopathic arthritis. Arthritis Rheum. 2000;43(4):765-774.

12. Fischer J, et al. IL-21+ CD4 $4^{+}$T helper cells co-expressing IFN-gamma and TNF-alpha accumulate in the joints of antinuclear antibody positive patients with juvenile idiopathic arthritis. Clin Immunol. 2020;217:108484.

13. Cosmi L, et al. Evidence of the transient nature of the Th17 phenotype of CD4+CD161+ T cells in the synovial fluid of patients with juvenile idiopathic arthritis. Arthritis Rheum. 2011;63(8):2504-2515.

14. Nistala K, et al. Th17 plasticity in human autoimmune arthritis is driven by the inflammatory environment. Proc Natl Acad Sci US A. 2010;107(33):14751-14756.

15. Mazzoni A, et al. Demethylation of the RORC2 and IL17A in human CD4+ T lymphocytes defines Th17 origin of nonclassic Th1 cells. J Immunol. 2015;194(7):3116-3126.

16. Basdeo SA, et al. Ex-Th17 (nonclassical Th1) cells are functionally distinct from classical Th1 and Th17 cells and are not constrained by regulatory T cells. J Immunol. 2017;198(6):2249-2259.

17. Annunziato F, et al. Phenotypic and functional features of human Th17 cells. J Exp Med. 2007;204(8):1849-1861.

18. Henderson LA, et al. Next-generation sequencing reveals restriction and clonotypic expansion of Treg cells in juvenile idiopathic arthritis. Arthritis Rheumatol. 2016;68(7):1758-1768.

19. Bending D, et al. Synovial regulatory T cells occupy a discrete TCR niche in human arthritis and require local signals to stabilize FOXP3 protein expression. J Immunol. 2015;195(12):5616-5624.

20. de Kleer IM, et al. CD4+CD25bright regulatory T cells actively regulate inflammation in the joints of patients with the remitting form of juvenile idiopathic arthritis. J Immunol. 2004;172(10):6435-6443.

21. Haufe $S$, et al. Impaired suppression of synovial fluid $C D 4+C D 25-T$ cells from patients with juvenile idiopathic arthritis by CD4+CD25+ Treg cells. Arthritis Rheum. 2011;63(10):3153-3162.

22. Wehrens EJ, et al. Functional human regulatory $\mathrm{T}$ cells fail to control autoimmune inflammation due to $\mathrm{PKB} / \mathrm{c}$-akt hyperactivation in effector cells. Blood. 2011;118(13):3538-3548.

23. Petrelli A, et al. Self-sustained resistance to suppression of CD8+ Teff cells at the site of autoimmune inflammation can be reversed by tumor necrosis factor and interferon- $\gamma$ blockade. Arthritis Rheumatol. 2016;68(1):229-236.

24. Bending D, et al. Hypomethylation at the regulatory $\mathrm{T}$ cell-specific demethylated region in CD25hi T cells is decoupled from FOXP3 expression at the inflamed site in childhood arthritis. J Immunol. 2014;193(6):2699-2708.

25. Cosmi L, et al. Human interleukin 17-producing cells originate from a CD161+CD4+ T cell precursor. J Exp Med. 2008;205(8):1903-1916.

26. Thornton AM, et al. Expression of Helios, an Ikaros transcription factor family member, differentiates thymic-derived from peripherally induced Foxp3+ T regulatory cells. J Immunol. 2010;184(7):3433-3441.

27. Himmel ME, et al. Helios+ and Helios- cells coexist within the natural FOXP3+ T regulatory cell subset in humans. J Immunol. 2013;190(5):2001-2008.

28. Henderson LA, et al. Th17 reprogramming of T cells in systemic juvenile idiopathic arthritis. JCI Insight. 2020;5(6):132508.

29. Hill JA, et al. Foxp3 transcription-factor-dependent and -independent regulation of the regulatory $\mathrm{T}$ cell transcriptional signature. Immunity. 2007;27(5):786-800.

30. Ferraro A, et al. Interindividual variation in human T regulatory cells. Proc Natl Acad Sci U S A. 2014;111(12):E1111-E1120.

31. Kmieciak M, et al. Human T cells express CD25 and Foxp3 upon activation and exhibit effector/memory phenotypes without any regulatory/suppressor function. J Transl Med. 2009;7:89.

32. Bradley $\mathrm{A}$, et al. Elucidating $\mathrm{T}$ cell activation-dependent mechanisms for bifurcation of regulatory and effector $\mathrm{T}$ cell differentiation by multidimensional and single-cell analysis. Front Immunol. 2018;9:1444.

33. Zemmour D, et al. Single-cell gene expression reveals a landscape of regulatory T cell phenotypes shaped by the TCR. Nat Immunol. 2018;19(3):291-301. 
34. Zheng Y, et al. Role of conserved non-coding DNA elements in the Foxp3 gene in regulatory T-cell fate. Nature. 2010;463(7282):808-812.

35. Samstein RM, et al. Foxp3 exploits a pre-existent enhancer landscape for regulatory $\mathrm{T}$ cell lineage specification. Cell. 2012;151(1):153-166.

36. Rao DA, et al. Pathologically expanded peripheral T helper cell subset drives B cells in rheumatoid arthritis. Nature. 2017;542(7639):110-114

37. Linterman MA, et al. Foxp3+ follicular regulatory T cells control the germinal center response. Nat Med. 2011;17(8):975-982.

38. Schneider WM, et al. Interferon-stimulated genes: a complex web of host defenses. Annu Rev Immunol. 2014;32:513-545.

39. Grose RH, et al. Comparison of blood and synovial fluid th17 and novel peptidase inhibitor 16 Treg cell subsets in juvenile idiopathic arthritis. JRheumatol. 2012;39(10):2021-2031.

40. Mahendra A, et al. Th1 and Th17 predominance in the enthesitis-related arthritis form of juvenile idiopathic arthritis. JRheumatol. 2009;3(8):1730-1736.

41. Maggi L, et al. Th17 and Th1 lymphocytes in oligoarticular juvenile idiopathic arthritis. Front Immunol. 2019;10:450.

42. Fergusson JR, et al. CD161 defines a transcriptional and functional phenotype across distinct human T cell lineages. Cell Rep . 2014;9(3):1075-1088.

43. Overacre-Delgoffe AE, et al. Interferon- $\gamma$ drives Treg fragility to promote anti-tumor immunity. Cell. 2017;169(6):1130-1141.

44. Duhen T, et al. Functionally distinct subsets of human FOXP3+ Treg cells that phenotypically mirror effector Th cells. Blood. 2012;119(19):4430-4440.

45. Mason GM, et al. Phenotypic complexity of the human regulatory T cell compartment revealed by mass cytometry. J Immunol. 2015;195(5):2030-2037.

46. Koch MA, et al. The transcription factor T-bet controls regulatory T cell homeostasis and function during type 1 inflammation Nat Immunol. 2009;10(6):595-602.

47. Koch MA, et al. T-bet(+) Treg cells undergo abortive Th1 cell differentiation due to impaired expression of IL-12 receptor beta2 Immunity. 2012;37(3):501-510.

48. Levine AG, et al. Stability and function of regulatory T cells expressing the transcription factor T-bet. Nature. 2017;546(7658):421-425

49. Charbonnier LM, et al. Control of peripheral tolerance by regulatory T cell-intrinsic Notch signaling. Nat Immunol. 2015;16(11):1162-1173.

50. Seddiki N, et al. Expression of interleukin (IL)-2 and IL-7 receptors discriminates between human regulatory and activated T cells. J Exp Med. 2006;203(7):1693-1700.

51. Kunicki MA, et al. Identity and diversity of human peripheral Th and T regulatory cells defined by single-cell mass cytometry. J Immunol. 2018;200(1):336-346.

52. Baecher-Allan C, et al. MHC class II expression identifies functionally distinct human regulatory T cells. J Immunol. 2006;176(8):4622-4631.

53. Duurland CL, et al. CD161(+) Tconv and CD161(+) Treg share a transcriptional and functional phenotype despite limited overlap in TCR $\beta$ repertoire. Front Immunol. 2017;8:103.

54. Pesenacker AM, et al. CD161 defines the subset of FoxP3+ T cells capable of producing proinflammatory cytokines. Blood 2013;121(14):2647-2658.

55. Rossetti M, et al. TCR repertoire sequencing identifies synovial Treg cell clonotypes in the bloodstream during active inflammation in human arthritis. Ann Rheum Dis. 2017;76(2):435-441.

56. Magen A, et al. Single-cell profiling defines transcriptomic signatures specific to tumor-reactive versus virus-responsive $\mathrm{CD}_{4}^{+} \mathrm{T}$ cells. Cell Rep. 2017;29(10):3019-3032.

57. Takeuchi A, Saito T. CD4 CTL, a cytotoxic subset of CD4+ T cells, their differentiation and function. Front Immunol. 2017;8:194

58. Appay V, et al. Characterization of CD4(+) CTLs ex vivo. J Immunol. 2002;168(11):5954-5958.

59. Brien JD, et al. West Nile virus-specific CD4 T cells exhibit direct antiviral cytokine secretion and cytotoxicity and are sufficient for antiviral protection. J Immunol. 2008;181(12):8568-8575.

60. Zhang JY, et al. Single-cell landscape of immunological responses in patients with COVID-19. Nat Immunol. 2020;21(9):1107-1118.

61. Meckiff BJ, et al. Imbalance of regulatory and cytotoxic SARS-CoV-2-reactive CD4 ${ }^{+} \mathrm{T}$ cells in COVID-19. Cell. 2020;183(5):1340-1353.

62. Picelli S, et al. Smart-seq2 for sensitive full-length transcriptome profiling in single cells. Nat Methods. 2013;10(11):1096-1098.

63. Dobin A, et al. STAR: ultrafast universal RNA-seq aligner. Bioinformatics. 2013;29(1):15-21.

64. Liao Y, et al. featureCounts: an efficient general purpose program for assigning sequence reads to genomic features. Bioinformatics. 2014;30(7):923-930

65. Patro R, et al. Salmon provides fast and bias-aware quantification of transcript expression. Nat Methods. 2017;14(4):417-419.

66. Love MI, et al. Moderated estimation of fold change and dispersion for RNA-seq data with DESeq2. Genome Biol. 2014;15(12):550.

67. Yu G, et al. clusterProfiler: an R package for comparing biological themes among gene clusters. OMICS. 2012;16(5):284-287.

68. Jassal B, et al. The reactome pathway knowledgebase. Nucleic Acids Res. 2020;48(D1):D498-D503.

69. Stoeckius M, et al. Cell Hashing with barcoded antibodies enables multiplexing and doublet detection for single cell genomics. Genome Biol. 2018;19(1):224.

70. Zheng GX, et al. Massively parallel digital transcriptional profiling of single cells. Nat Commun. 2017;8:14049.

71. Stuart T, et al. Comprehensive integration of single-cell data. Cell. 2019;177(7):1888-1902.

72. Oksanen J, et al. Vegan: community ecology package. R package version 2.5-7. https://CRAN.R-project.org/package=vegan . Accessed August 18, 2021. 